\title{
The chemistry of mercapto- and thione- substituted 1,2,4-triazoles and their utility in heterocyclic synthesis
}

\author{
Raafat M. Shaker \\ Chemistry Department, Faculty of Science, El-Minya University, El-Minya, Egypt; \\ E-mail: rmshaker@yahoo.com
}

\begin{abstract}
The synthesis and reactions of mercapto- and thione-substituted 1,2,4-triazoles are comprehensively reviewed.

Keywords: $\quad$ Mercapto-1,2,4-triazoles, 1,2,4-triazolethiones, thiazolotriazoles, triazolothiadiazoles, triazolothiazines, triazolothiazepines, triazolothiadiazines
\end{abstract}

\section{Table of Contents}

1. Introduction

2. Synthetic approaches

2.1. Synthesis of 2,4-dihydro-3H-mercapto/thione-1,2,4-triazoles

2.2. Synthesis of 4-alkyl/aryl-mercapto/thione-1,2,4-triazoles

2.3. Synthesis of 2,4-dialkyl/aryl-1,2,4-triazolethiones

2.4. Synthesis of 1,2,4-triazole-3,5-dithiones

2.5. Synthesis of fused mercapto/thione-1,2,4-triazole heterocycles

3. Chemical reactivity

3.1. Alkylation and arylation

3.2. Synthesis of monosulfides and disulfides

3.3. Synthesis of mannich base derivatives

3.4. Synthesis of thiazolotriazoles

3.5. Synthesis of triazolothiadiazoles

3.6. Synthesis of triazolothiazines

3.7. Synthesis of triazolothiazepine

3.8. Synthesis of triazolothiadiazines

3.9. Miscellaneous reactions

4. Conclusions

5. References 


\section{Introduction}

In the last few decades, the chemistry of 1,2,4-triazoles and their fused heterocyclic derivatives has received considerable attention owing to their synthetic and effective biological importance. For example, a large number of 1,2,4-triazole-containing ring system have been incorporated into a wide variety of therapeutically interesting drug candidates including anti-inflammatory, CNS stimulants sedatives, antianxiety, antimicrobial agents ${ }^{1,2}$ and antimycotic activity such as fluconazole, intraconazole, voriconazole. ${ }^{3,4}$ Also, there are known drugs containing the 1,2,4triazole group e.g. Triazolam ${ }^{5}$, Alprazolam ${ }^{6}$, Etizolam $^{7}$, and Furacylin ${ }^{8}$. Moreover, sulphurcontaining heterocycles represent an important group of sulphur compounds that are promising for use in practical applications. Among these heterocycles, the mercapto- and thione-substituted 1,2,4-triazole ring systems A-C (Figure 1) have been well studied and so far a variety of biological activities have been reported for a large number of their derivatives, such as antibacterial $^{9-12}$, antifungal ${ }^{13,14}$, antitubercular ${ }^{15}$, antimycobacterial ${ }^{16}$, anticancer ${ }^{17,18}$, diuretic ${ }^{19,20}$, and hypoglycemic ${ }^{21}$ properties.<smiles>[R]c1n[nH]c(=S)n1[R]</smiles>

A

$$
\mathrm{R}=\mathrm{H} \text {, alkyl, aryl }
$$<smiles>[R]c1nnc(S)n1[R]</smiles>

$\mathrm{R}$<smiles>[R]c1nn([R2])c(=S)n1[R]</smiles>

B<smiles>[R]n1c(=S)[nH][nH]c1=S</smiles>

C

$\mathrm{R}=\mathrm{R}^{1}=\mathrm{R}^{2}=$ alkyl, aryl

\section{Figure 1}

In addition to these important biological applications, mercapto-1,2,4-triazoles are also of great utility in preparative organic chemistry, for example, in the present of various reagents, undergo different types of reactions to yield other heterocyclic compounds, e.g., thiazolotriazoles, triazolothiadiazoles, triazolothiazines, triazolothiazepines and triazolothiadiazines. Recently ${ }^{22}$, we have reviewed the chemistry of the 4-amino-1,2,4-triazole-3thiones $\left(\mathrm{A}, \mathrm{R}=\mathrm{NH}_{2}\right)$.

In the present review article the most common and useful procedures for the preparation of the mercapto- and thione-substituted 1,2,4-triazole and their utility for the synthesis of well known heterocyclic ring systems are compiled and discussed, therewith the most attention is paid to reports published within the last 50 years. 


\section{Synthetic approaches}

\subsection{Synthesis of 2,4-dihydro-3H-mercapto/thione-1,2,4-triazoles}

The reaction of carboxylic acid chlorides 1 and thiosemicarbazide gave 2, which without purification were cyclized in alkaline media to yield the corresponding 2,4-dihydro-3H--1,2,4triazole-3-thiones $\mathbf{3}^{23-37}$ (Scheme 1).

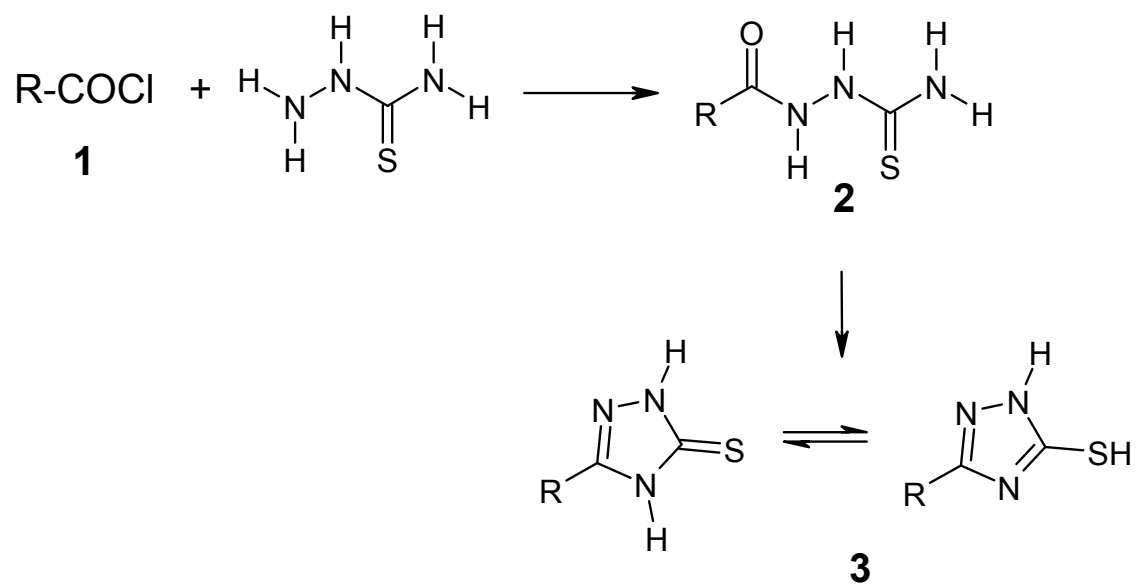

$$
\begin{aligned}
\mathrm{R}= & \mathrm{H}, \mathrm{CH}_{3}, \mathrm{CF}_{3}, \mathrm{C}_{6} \mathrm{~F}_{13}, \text { 1-adamentyl, } \mathrm{C}_{6} \mathrm{H}_{5}, 4-\mathrm{CH}_{3} \mathrm{C}_{6} \mathrm{H}_{4}, 4-\mathrm{ClC}_{6} \mathrm{H}_{4}, 4-\mathrm{BrC}_{6} \mathrm{H}_{4}, \\
& \text { 2-, 3-, and 4- } \mathrm{CH}_{3} \mathrm{OC}_{6} \mathrm{H}_{4}, 2-, \text { 3-, and 4- } \mathrm{FC}_{6} \mathrm{H}_{4}, 4-\mathrm{O}_{2} \mathrm{NC}_{6} \mathrm{H}_{4}, 2,4-(\mathrm{Cl})_{2}-5-\mathrm{F}-\mathrm{C}_{6} \mathrm{H}_{2}, \\
& \text { 2,3,5-( }(\mathrm{F})_{3}-4-\mathrm{CH}_{3} \mathrm{OC}_{6} \mathrm{H}, 4-\mathrm{ClC}_{6} \mathrm{H}_{4} \mathrm{SO}_{2} \mathrm{CH}_{2} \mathrm{CH}_{2}, 1,3 \text {-benzodioxo-5-yl }
\end{aligned}
$$

\section{Scheme 1}

The triazoles $4^{38}$ were also prepared by reaction of acid halides with a lead (II) thiocyanate and hydrazine hydrate $(15 \%)$ in a solvent at -70 to $+200^{\circ} \mathrm{C}$ (Scheme 2).

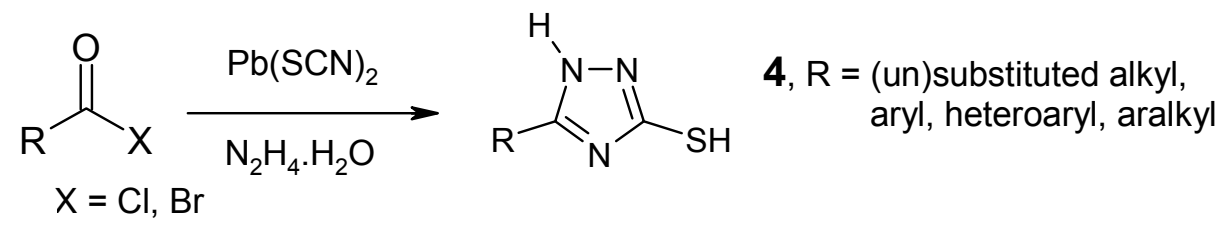

\section{Scheme 2}

In addition, the triazolethiones $6^{39}$ can be prepared readily from the thermolysis of thiosemicarbazones 5 (Scheme 3). 


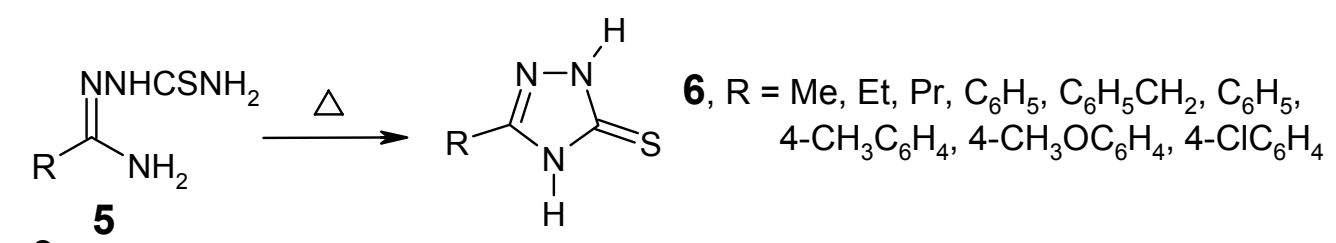

e 3.

\section{Scheme 3}

Goswami et al. $^{40}$ reported that the oxidative cyclization of 1-(2,4-dichloro-benzoyl)thiosemicarbazide (7) gives the 3-(2,4-dichlorophenyl)-1H-1,2,4-triazole-5-thiol (8) (Scheme 4). Compound $\mathbf{8}$ exhibited antimicrobial activities against B. Cereus, Esch. Coli and P. Salanarium.

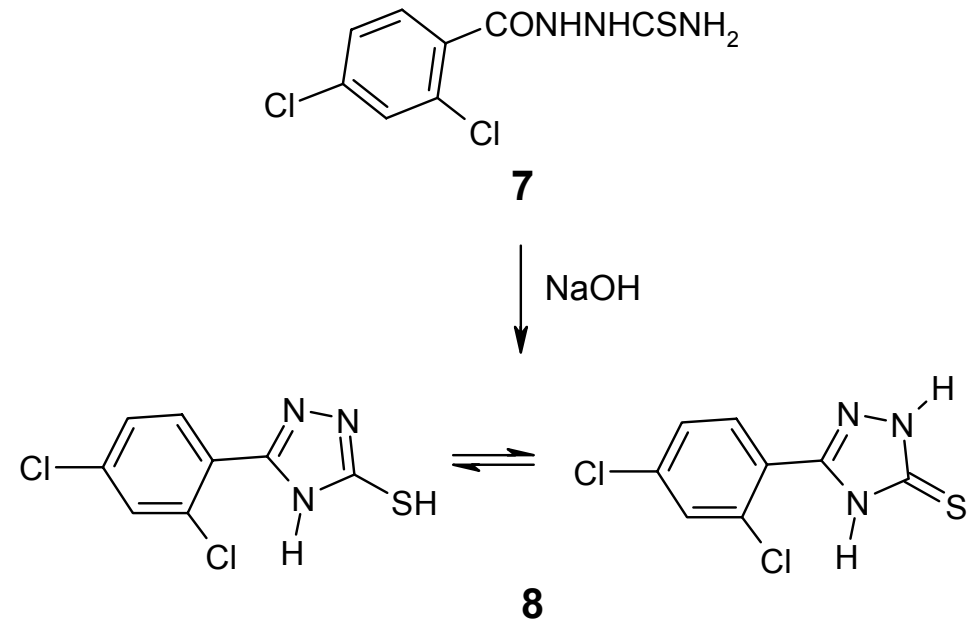

\section{Scheme 4}

Moreover, the 1,2,4-triazole-5-thiones $\mathbf{9}^{41}$ and $\mathbf{1 0}^{42}$ were prepared by cyclization of the corresponding thiosemicarbazide (Figure 2). Compound 9 possessed anti-inflammatory activity $^{41}$.<smiles>CC(C)Cc1ccc(C(C)c2n[nH]c(=S)[nH]2)cc1</smiles><smiles>[R][CH]OC(C)=O</smiles>

Figure 2 
The bis(5-mercapto-4H-1,2,4-triazol-3-yl)alkanes $\mathbf{1 1}^{43}$ can also be obtained by the directly treating the corresponding aliphatic dicarboxylic acids with thiosemicarbazide (Scheme 5).<smiles>C=CCc1nnc(S)n1[Hg]c1nnc(S)n1[TlH]</smiles><smiles>Cc1nn([TlH])c(=S)[nH]1</smiles>

\section{Scheme 5}

Starting from pyroglutamic esters, 1,2,4-triazole-3-thiones $\mathbf{1 2}^{\mathbf{4 4}}$, bonded to a pyrrolidinone ring were synthesized (Figure 3).<smiles>[R]N1C(=O)CCC1c1n[nH][nH]c1=S</smiles>

12, $\mathrm{R}=\mathrm{H}, \mathrm{C}_{6} \mathrm{H}_{5} \mathrm{CH}_{2}$

\section{Figure 3}

5-Furan-2-yl-4H-1,2,4-triazole-3-thiol (14) was prepared by the reaction of the appropriate 2-furoyl-thiosemicarbazide (13) and potassium hydroxide in ethanol for 3 hours under reflux, followed by acidification with acetic acid ${ }^{45,46}$ (Scheme 6). It has been reported that the crystal structure of $\mathbf{3}$ corresponded to the thione form, but they showed thiol-thione tautomerism in solution $^{47}$.<smiles>CCOCc1cccc(C(=O)NNC(=S)NNC(=O)c2ccco2)c1</smiles>

13<smiles>S=c1[nH]nc(-c2ccco2)[nH]1</smiles><smiles>C1=CC=C1</smiles>

14<smiles>Sc1nc(-c2ccco2)n[nH]1</smiles>

\section{Scheme 6}

Condensation of carbamate $\mathbf{1 5}$ with thiosemicarbazide in boiling pyridine via initial nucleophilic attack of the amino group to the ester carbonyl without attack at the carbonyl of the pyrazine ring followed by cyclization to give triazolylquinoxaline $\mathbf{1 6}^{48,49}$ (Scheme 7). 
<smiles>CC(=O)Nc1nc2ccccc2[nH]c1=O</smiles>

15

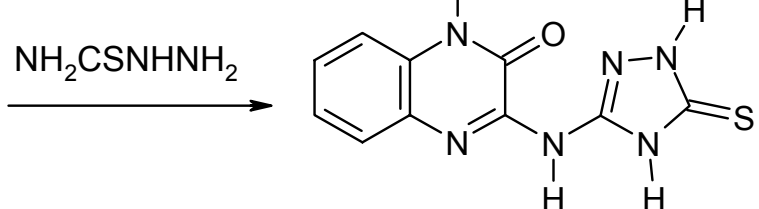

16

\section{Scheme 7}

5-(4,6-Diphenyl-pyrimidin-2-yl)-1,2,4-triazolin-3-thione (19) ${ }^{50}$ could be prepared either by base catalyzed cyclization of acylthiosemicarbazide 17 or by the reaction of methyl pyrimidin-2carboxylate $\mathbf{1 8}$ with thiosemicarbazide in the presence of $\mathrm{NaOH}$ (Scheme 8).<smiles>CC(=O)c1nc(-c2ccccc2)cc(-c2ccccc2)n1</smiles>

\section{Scheme 8}

Similarly, triazoles $\mathbf{2 0}$ and $\mathbf{2 1}$ were obtained by the cyclization of the corresponding thiosemicarbazides in alkaline medium ${ }^{51,52}$ (Figure 4).<smiles>Sc1nnc(Cc2c[nH]c3ccccc23)[nH]1</smiles>

20<smiles>Cn1c(C(=O)O)c(-c2n[nH]c(=S)[nH]2)c2ccccc21</smiles>

21

\section{Figure 4}

When the reaction of hydrazide 22 with ammonium thiocyanate was carried out in boiling hydrochloric acid, the 1-(2-benzothiazolylcarbonyl)thiosemicarbazide (23) was obtained. Upon heating of the later compound with sodium hydroxide, it underwent intramolecular cyclization to give 3-(2-benzothiazolyl)-1,2,4-triazoline-5-thione (24) ${ }^{53}$ (Scheme 9). 
<smiles>CC(C)c1ccc2sc(-c3n[nH]c(=S)n3[TlH])nc2c1</smiles>

22

23

24

\section{Scheme 9}

\subsection{Synthesis of 4-alkyl/aryl-mercapto/thione-1,2,4-triazoles}

The cyclodehydration of thiosemicarbazides 25 in alkaline medium afforded 4-alkyl/aryl-1,2,4triazoline-3-thiones $\mathbf{2 6}^{36,38,54-63}$ (Scheme 10). It has been reported that the compounds 26 exist mainly in a thione form ${ }^{62}$.

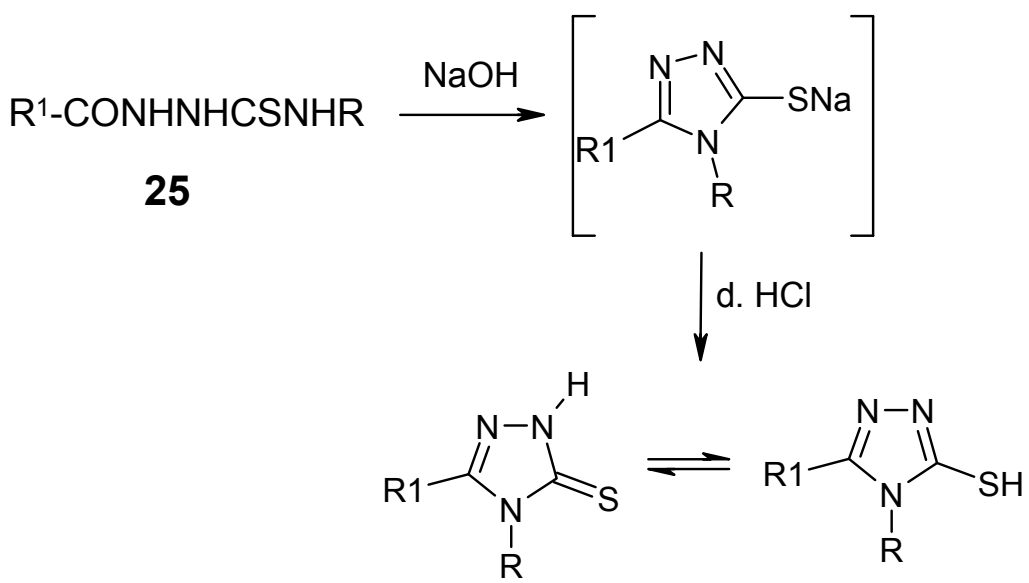

26

$\mathrm{R}=\mathrm{CH}_{3}, \mathrm{C}_{2} \mathrm{H}_{5}, \mathrm{C}_{6} \mathrm{H}_{11}, \mathrm{CH}_{2} \mathrm{CH}_{2} \mathrm{OH}, \mathrm{CH}_{2} \mathrm{CO}_{2} \mathrm{C}_{2} \mathrm{H}_{5}, \mathrm{C}_{6} \mathrm{H}_{5}, \mathrm{C}_{6} \mathrm{H}_{5} \mathrm{CH}_{2}, 4-\mathrm{CH}_{3} \mathrm{O}-\mathrm{C}_{6} \mathrm{H}_{4}$;

$\mathrm{R}^{1}=\mathrm{H}, \mathrm{Me}, \mathrm{Et}, \mathrm{Pr}, \mathrm{Bu}, 3-\mathrm{C}_{5} \mathrm{H}_{4} \mathrm{~N}, \mathrm{C}_{6} \mathrm{H}_{5}, 4-\mathrm{BrC}_{6} \mathrm{H}_{4} \mathrm{SCH}_{2}, 4-\mathrm{CH}_{3} \mathrm{C}_{6} \mathrm{H}_{4} \mathrm{SCH}_{2} \mathrm{CH}_{2}$, 4- $\mathrm{ClC}_{6} \mathrm{H}_{4} \mathrm{SO}_{2} \mathrm{CH}_{2} \mathrm{CH}_{2}, 4-\mathrm{BrC}_{6} \mathrm{H}_{4} \mathrm{SO}_{2} \mathrm{CH}_{2}, 4-\mathrm{ClC}_{6} \mathrm{H}_{4}, 2-\mathrm{FC}_{6} \mathrm{H}_{4}$,

\section{Scheme 10}

Lin et al. $^{64}$ reported that the 4-substituted-4H-1,2,4-triazole-3-thiols 27 were prepared by the condensation of 4-substituted-3-thiosemicarbazides with dimethylformamide dimethyl acetal or dimethylacetamide dimethyl acetal (Figure 5). 


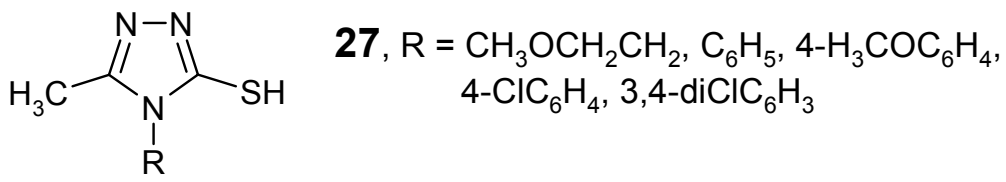

\section{Figure 5}

On the other hand, 4-alkyl/aryl-5-nonanoyl/octadecanoyl-2,4-dihydro-3H-1,2,4-triazoline3-thiones were synthesized as potential antimicrobial agents. The course of synthesis included the reaction of nonanoyl/octadecanoyl hydrazine with selected alkyl/aryl isothiocyanates. The prepared thiosemicarbazides gave by cyclization the required 1,2,4-triazoles $\mathbf{2 8}^{65}$ (Figure 6).

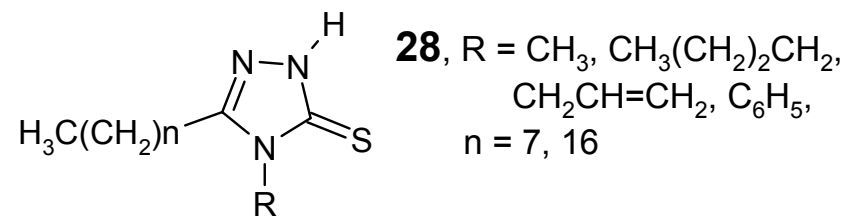

\section{Figure 6}

Moreover, a number of 2-hydroxycycloalkyl-substituted 1,2,4-triazoles 29 and $\mathbf{3 0}$ were prepared by different methods from cis- and trans-2-hydroxy-1-cycloalkane-carbohydrazides and their isocyanate or isothiocyanate adducts ${ }^{66}$ (Figure 7).<smiles>[R]n1c(S)nnc1C1CCCC1O</smiles>

29<smiles>[R]n1c(S)nnc1C1CCCCC1O</smiles>

30

Figure 7

The 1-(2,4-dichlorobenzoyl)-4-arylthiosemicarbazides 31 on oxidative cyclization with $1 N$ $\mathrm{NaOH}$ solution under reflux resulted in their corresponding 1,2,4-triazole-5-thiols $\mathbf{3 2}^{40}$ (Scheme 11).

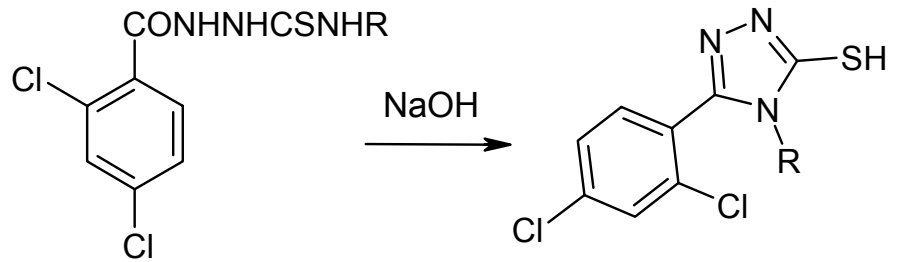

32, $\mathrm{R}=\mathrm{C}_{6} \mathrm{H}_{5} \mathrm{CH}_{2}, \mathrm{C}_{6} \mathrm{H}_{5}, 4-\mathrm{BrC}_{6} \mathrm{H}_{4}$, 2-, 3-, and 4- $\mathrm{ClC}_{6} \mathrm{H}_{4}$, 2-, and 4- $\mathrm{CH}_{3} \mathrm{C}_{6} \mathrm{H}_{4}$, 2-, and 4- $\mathrm{CH}_{3} \mathrm{OC}_{6} \mathrm{H}_{4}$,

31

\section{Scheme 11}


The 1,2,4-triazoles $\mathbf{3 4}^{23,37,67,68}$ and $\mathbf{3 5}^{57}$ have also been synthesized by the condensation and cyclization of the corresponding thiosemicarbazides 33a-d in $\mathrm{NaOH}$ solution (Scheme 12).

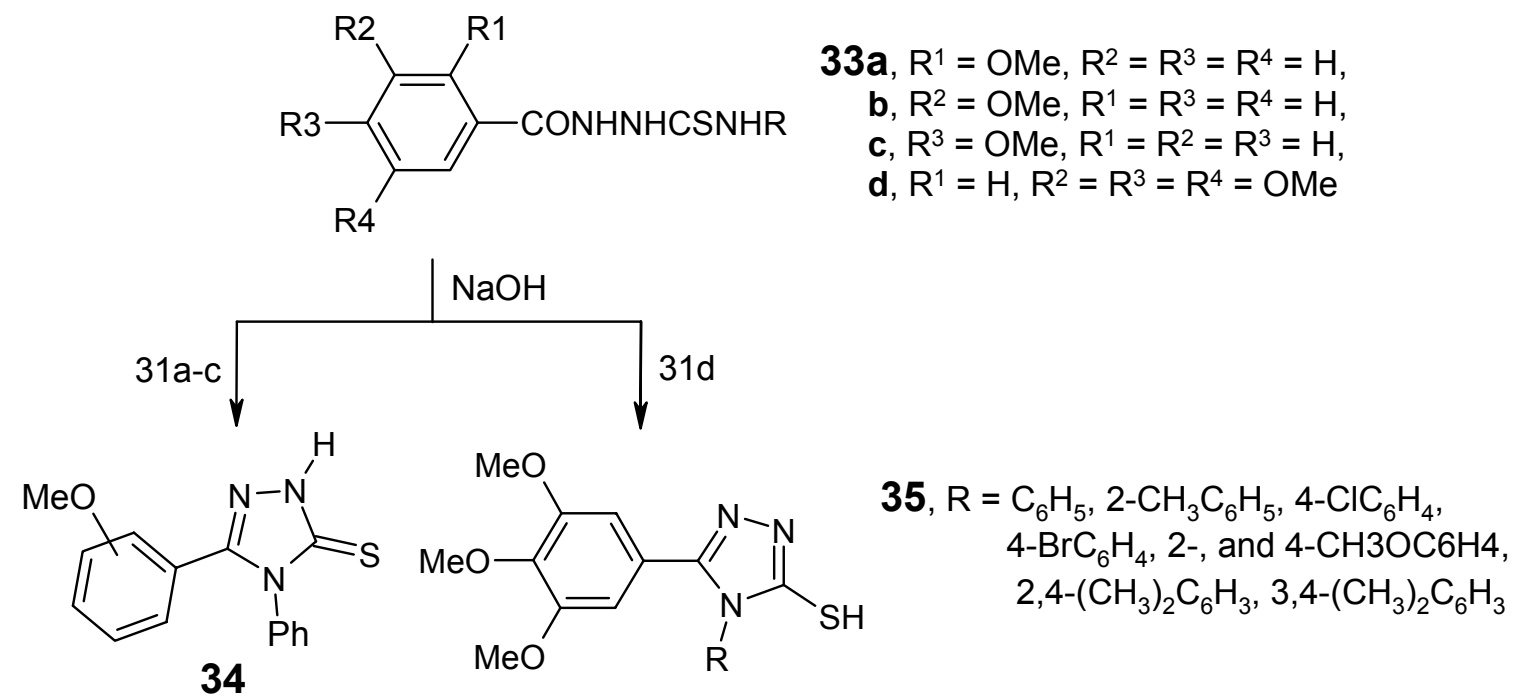

\section{Scheme 12}

When compounds 36 were refluxed in $2 M$ sodium hydroxide solutions for about 4 hours, they produced 4-allyl-5-aryl-1,2,4-triazoles 37 in good yields ${ }^{69,70,71}$ (Scheme 13). While compound $\mathbf{3 7}$ may exist in thione-thiol tautomeric forms, the authors reported that in this case the thione structures dominate in the solid state. Compound $\mathbf{3 7}$ showed inhibitory effects against Escherichia coli, Bacillus subtilis, Salmonella enteritidis, Staphylococcus aureus, Aspergillus niger and Candida albicans ${ }^{71}$.<smiles>C=CCNC(=S)NNC(=O)Br</smiles>

36<smiles>C=CCn1c(S)nnc1[Bi]</smiles>

37, $\mathrm{Ar}=\mathrm{C}_{6} \mathrm{H}_{5}, 2-\mathrm{OHC}_{6} \mathrm{H}_{4}, 4-\mathrm{OHC}_{6} \mathrm{H}_{4}$, 2-OH-5- $-\mathrm{ClC}_{6} \mathrm{H}_{4}, 4-\mathrm{C}_{2} \mathrm{H}_{5} \mathrm{OC}_{6} \mathrm{H}_{4} \mathrm{CH}_{2}$

13.

\section{Scheme 13}

Refluxing the corresponding thiosemicarbazides in alkaline medium performed the $1,2,4-$ triazolethiones $\mathbf{3 8}^{14,72}$ and $\mathbf{3 9}^{73}$ (Figure 8). 


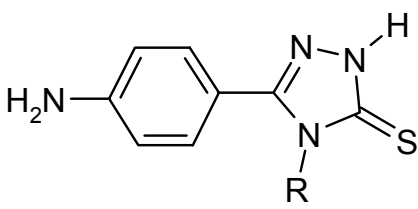

38, $\mathrm{R}=\mathrm{CH}_{3} \mathrm{CH}_{2}, \mathrm{CH}_{2}-\mathrm{CH}=\mathrm{CH}_{2}, \mathrm{C}_{6} \mathrm{H}_{5}$

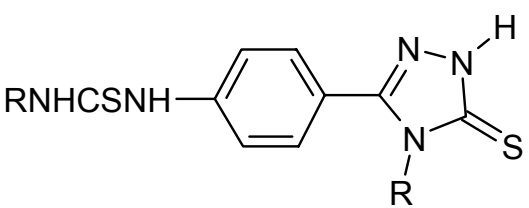

39, $\mathrm{R}=\mathrm{C}_{6} \mathrm{H}_{5}, 2-$ and $4-\mathrm{CH}_{3} \mathrm{C}_{6} \mathrm{H}_{4}$,

$\left(\mathrm{CH}_{3}\right)_{2} \mathrm{CH}, \mathrm{CH}_{3}-\left(\mathrm{CH}_{2}\right)_{3}-$

\section{Figure 8}

Substituted 1,4-Dihydro-3-(3-hydroxy-2-naphthyl)-5H-1,2,4-triazoline-5-thiones $\quad \mathbf{4 1}^{18,74}$ were obtained by the cyclization of thiosemicarbazides 40 in sodium hydroxide solution followed by treatment of the reaction mixture with dil. $\mathrm{HCl}$ at $0^{\circ} \mathrm{C}$ (Scheme 14).

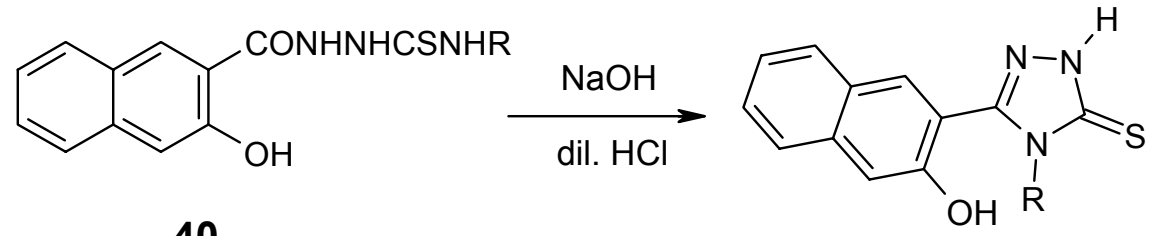

40

$$
\begin{aligned}
& \text { 41, } \mathrm{R}=\mathrm{C}_{2} \mathrm{H}_{5}, \mathrm{C}_{3} \mathrm{H}_{7}, \mathrm{C}_{6} \mathrm{H}_{11}, \mathrm{C}_{6} \mathrm{H}_{5}, \mathrm{C}_{6} \mathrm{H}_{5} \mathrm{CH}_{2} \text {, } \\
& \text { 4- } \mathrm{CH}_{3} \mathrm{C}_{6} \mathrm{H}_{4}, 2-\text {, 3- and 4- } \mathrm{FC}_{6} \mathrm{H}_{4} \text {, }
\end{aligned}
$$

\section{Scheme 14}

4,5-Disubstituted-2,4-dihydro-1,2,4-triazole-3-thiones $\mathbf{4 3}^{38}$ were obtained by the action of primary amines on thiosemicarbazones of ester $\mathbf{4 2}$ (Scheme 15).

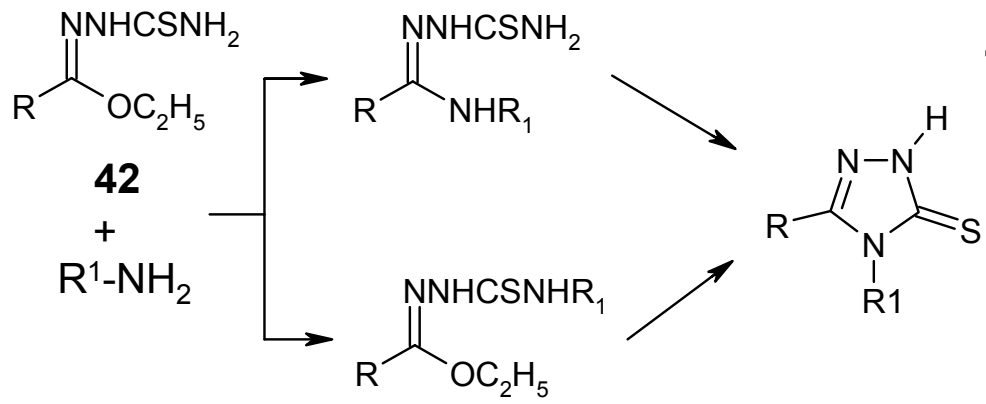

$$
\text { 43, } \begin{aligned}
\mathrm{R}= & \mathrm{CH}_{3}, \mathrm{C}_{2} \mathrm{H}_{5}, n-\mathrm{C}_{3} \mathrm{H}_{7}, \\
& \mathrm{C}_{6} \mathrm{H}_{5}, 4-\mathrm{CH}_{3} \mathrm{C}_{6} \mathrm{H}_{4}, \\
& 4-\mathrm{CH}_{3} \mathrm{OC}_{6} \mathrm{H}_{4}, \\
& 4-\mathrm{Cl}_{-} \mathrm{C}_{6} \mathrm{H}_{4}, \mathrm{C}_{6} \mathrm{H}_{5} \mathrm{CH}_{2}, \\
\mathrm{R}^{1}= & \text { cyclohexyl, } \mathrm{C}_{6} \mathrm{H}_{4} \mathrm{CH}_{2}, \\
& \mathrm{C}_{6} \mathrm{H}_{5}, 4-\mathrm{FC}_{6} \mathrm{H}_{4}, \\
& \text { 2-, and } 4-\mathrm{O}_{2} \mathrm{NC}_{6} \mathrm{H}_{4}, \\
& \text { 2-, and } 3-\mathrm{ClC}_{6} \mathrm{H}_{4},
\end{aligned}
$$

\section{Scheme 15}

5-Aryl-1,2-dihydro-2-(2-hydroxyethyl)-3H-1,2,4-triazole-3-thiones 44 were prepared from the reaction of 2-hydrazinoethanol with aroyl isothiocyanate in anhydrous benzene and in the presence of $p$-toluenesulfonic $\operatorname{acid}^{75}$ (Scheme 16). 


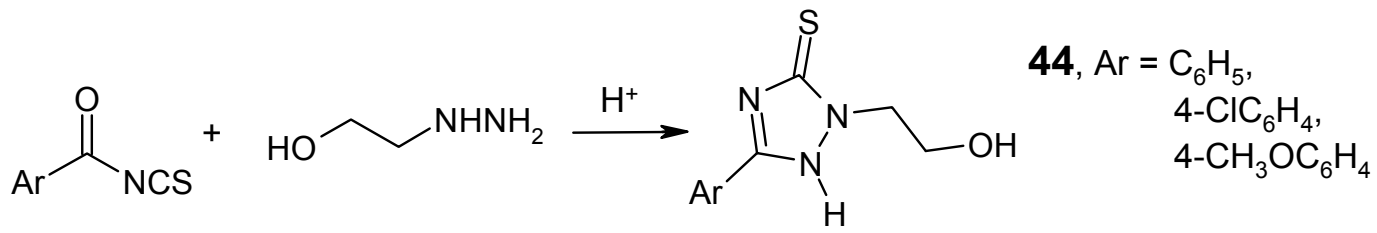

\section{Scheme 16}

The thiosemicarbazide $\mathbf{4 5}$ was cyclized with $2 \%$ or $10 \%$ solutions of sodium hydroxide to the corresponding 4-substituted-5-mercapto-1,2,4-triazole-3-acetic acid $\mathbf{4 6}^{\mathbf{7 6}}$ (Scheme 17). It has been reported that the compounds $\mathbf{4 6}$ exist mainly in a thiol form ${ }^{76}$.<smiles>[R]n1c(S)nnc1CC(=O)O</smiles>

45

$$
\text { 46, } \begin{aligned}
\mathrm{R}= & \mathrm{C}_{6} \mathrm{H}_{5}, 4-\mathrm{CH}_{3} \mathrm{C}_{6} \mathrm{H}_{4}, 3-, \text { and } 4-\mathrm{OCH}_{3} \mathrm{C}_{6} \mathrm{H}_{4} \text {, } \\
& 4-\mathrm{BrC}_{6} \mathrm{H}_{4}, 2,4\left(\mathrm{CH}_{3}\right)_{2} \mathrm{C}_{6} \mathrm{H}_{3}, \mathrm{CH}_{2} \mathrm{C}_{6} \mathrm{H}_{5}, \\
& \mathrm{C}_{6} \mathrm{H}_{11}, \mathrm{C}_{2} \mathrm{H}_{5}, \mathrm{C}_{4} \mathrm{H}_{9}, \mathrm{COOC}_{2} \mathrm{H}_{5}
\end{aligned}
$$

\section{Scheme 17}

The 1-(phenyl acetyl)-4-substituted thiosemicarbazides 47 on refluxing with $2 \mathrm{~N} \mathrm{NaOH}$ solution were cyclized into the corresponding 5-benzyl-4-aryl-4H-1,2,4-triazole-3-thiol $\mathbf{4 8}^{77}$ (Scheme 18).

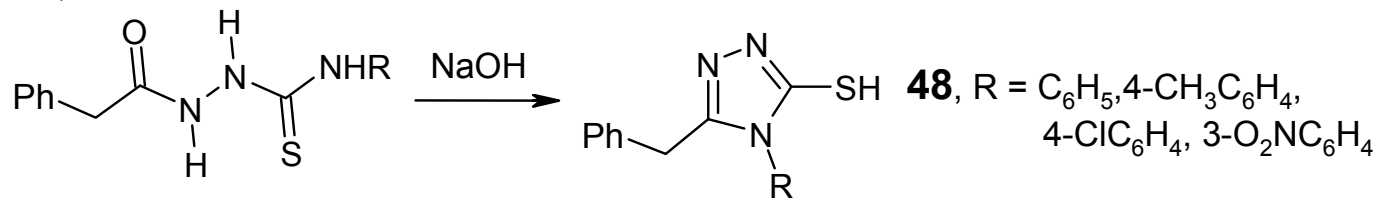

\section{7}

\section{Scheme 18}

4-(2-Halobenzyl)-1,2,4-triazole-3-thiones $\mathbf{5 2}$ were synthesized by refluxing the thiosemicarbazide $\mathbf{5 1}$ obtained from $o$-halobenzyl isothiocyanate $\mathbf{5 0}$ and an acid hydrazide $\mathbf{4 9}^{10,78}$ (Scheme 19). 


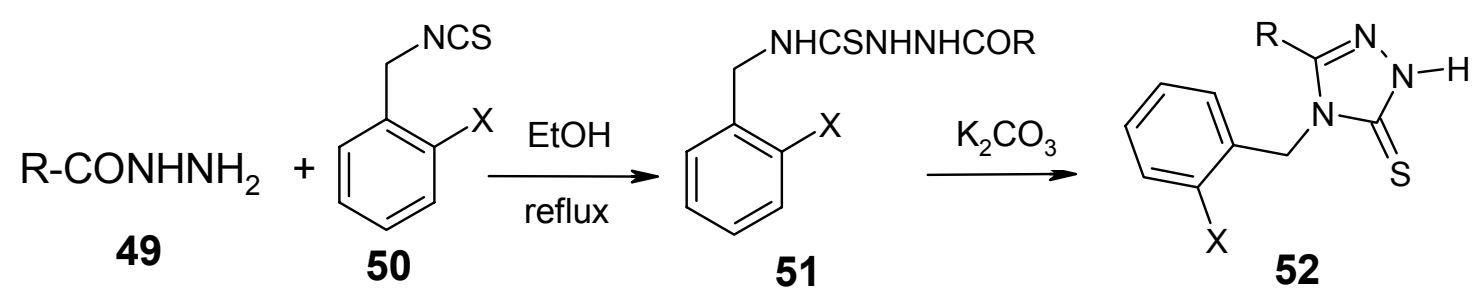
$\mathrm{R}=\mathrm{C}_{6} \mathrm{H}_{5}, 4-\mathrm{CH}_{3} \mathrm{C}_{6} \mathrm{H}_{4}, 4-\mathrm{CH}_{3} \mathrm{OC}_{6} \mathrm{H}_{4}, 2-\mathrm{CH}_{3} \mathrm{C}_{6} \mathrm{H}_{4}, 2-\mathrm{ClC}_{6} \mathrm{H}_{4}, \mathrm{C}_{6} \mathrm{H}_{5} \mathrm{CH}_{2} ; \quad \mathrm{X}=\mathrm{Cl}, \mathrm{Br}$

\section{Scheme 19}

Several 5-(1-naphthylmethyl)-4-aryl-s-triazole-3-thiols 53 were prepared as possible antiinflammatory agents ${ }^{79}$. Also, estradiol-17 $\alpha$-triazolines 54 were synthesized and tested in vitro for anabolic-catabolic activity and binding affinity to steroid receptors ${ }^{80}$ (Figure 9).

53, $\mathrm{R}=\mathrm{C}_{6} \mathrm{H}_{5}, 2-\mathrm{CH}_{3} \mathrm{C}_{6} \mathrm{H}_{4}$, 4- $-\mathrm{ClC}_{6} \mathrm{H}_{4}, 4-\mathrm{BrC}_{6} \mathrm{H}_{4}$, $2-\mathrm{H}_{3} \mathrm{COC}_{6} \mathrm{H}_{4}, 4-\mathrm{IC}_{6} \mathrm{H}_{4}$

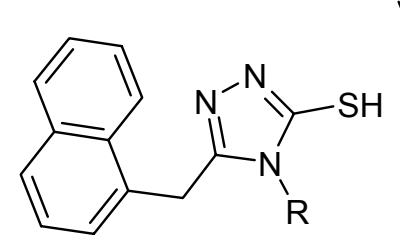

\section{Figure 9}

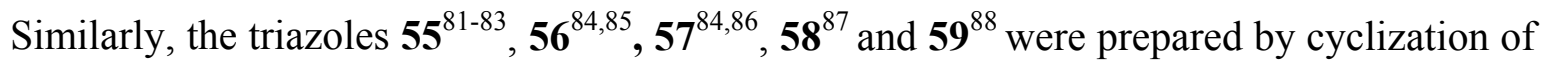
the corresponding 4-substituted-thiosemicarbazides in alkaline medium (Figure 10). Compound $57\left(\mathrm{R}=\mathrm{C}_{2} \mathrm{H}_{5}\right)$ showed interesting anti-inflammatory activity ${ }^{86}$. Also compound $58(\mathrm{R}=4-$ $\mathrm{ClC}_{6} \mathrm{H}_{4}$ ) showed significant antifungal and antibacterial activities ${ }^{87}$. 
<smiles>[R1]C(Oc1ccccc1)c1nn([IH])c(=S)n1[R]</smiles>

$$
55
$$

55, $\mathrm{R}=\mathrm{CH}_{3} \mathrm{CH}_{2}, \mathrm{C}_{6} \mathrm{H}_{5}$, $\mathrm{R}^{1}=\mathrm{H}, \mathrm{Cl}, \mathrm{CH}_{3}$,<smiles></smiles>
58, $\mathrm{R}=\mathrm{C}_{6} \mathrm{H}_{5}, 3-$, and 4- $\mathrm{ClC}_{6} \mathrm{H}_{4}, 4-\mathrm{CH}_{3} \mathrm{OC}_{6} \mathrm{H}_{4}$, 2-, 3-, and 4- $\mathrm{CH}_{3} \mathrm{C}_{6} \mathrm{H}_{4}$,<smiles>[R]n1c(COc2ccc3ccccc3c2)n[nH]c1=S</smiles>

57, $\mathrm{R}=\mathrm{CH}_{3}, \mathrm{C}_{2} \mathrm{H}_{5}$, $\mathrm{CH}_{2}-\mathrm{CH}=\mathrm{CH}_{2}$, $\mathrm{C}_{6} \mathrm{H}_{5}$<smiles>[R]n1c(S)nnc1COc1nnc(-c2ccccc2)c(-c2ccccc2)n1</smiles>

59, $\mathrm{R}=\mathrm{C}_{6} \mathrm{H}_{5}, 4-\mathrm{H}_{3} \mathrm{CC}_{6} \mathrm{H}_{4}, 4-\mathrm{ClC}_{6} \mathrm{H}_{4}$, $4-\mathrm{H}_{3} \mathrm{COC}_{6} \mathrm{H}_{4}, 4-\mathrm{H}_{3} \mathrm{CCH}_{2} \mathrm{OC}_{6} \mathrm{H}_{4}$,

\section{Figure 10}

The 1,2,4-triazoline-5-thiones $\mathbf{6 1} \mathbf{1}^{89}$ and $\mathbf{6 2}^{90}$ were synthesized by the cyclization of the corresponding thiosemicarbazide derivatives 60a,b in $\mathrm{NaOH}$ solution (Scheme 20). Compound $61\left(\mathrm{R}=\mathrm{C}_{2} \mathrm{H}_{5}, 4-\mathrm{CH}_{3} \mathrm{C}_{6} \mathrm{H}_{4}, 4-\mathrm{CH}_{3} \mathrm{OC}_{6} \mathrm{H}_{4}\right)$ showed antifungal activity against some species belonging to Trichophyton spp. known as the causative agents of superficial mycoses ${ }^{89}$.

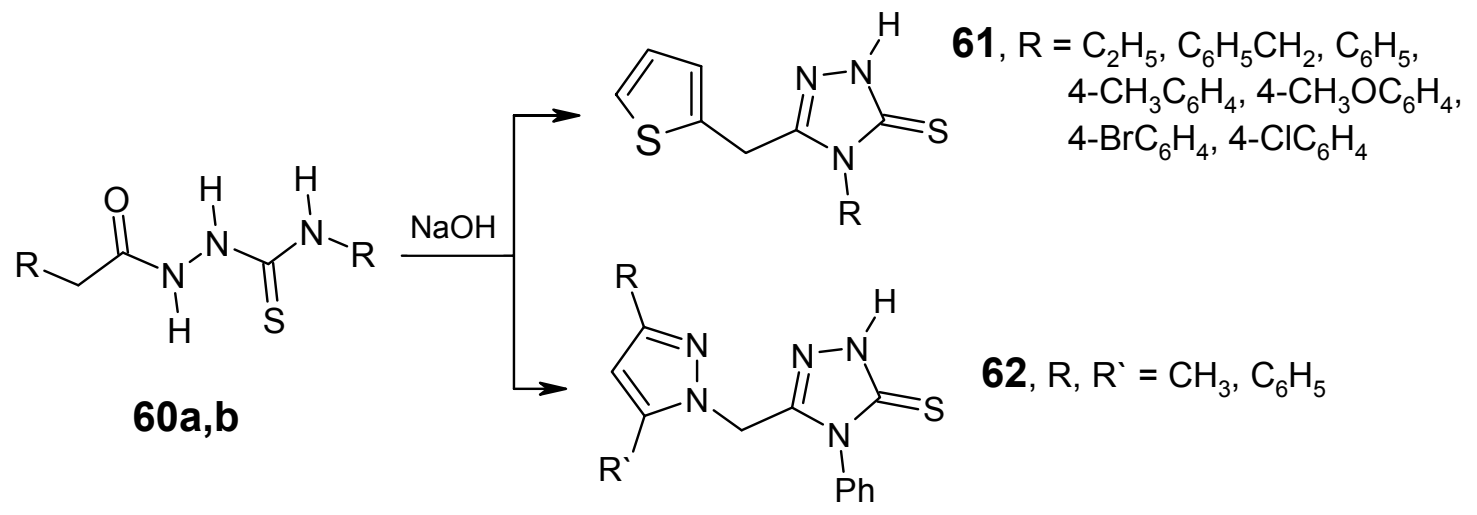

\section{Scheme 20}

Also, the triazolethiones $\mathbf{6 3}{ }^{91}, \mathbf{6 4}^{92,93}$ and $\mathbf{6 5}^{94}$ were synthesized from the corresponding 4substituted thiosemicarbazides in alkaline medium (Figure 11). Compound $63\left(\mathrm{R}=\mathrm{CH}_{3}\right.$, $\mathrm{CH}_{2} \mathrm{C}_{6} \mathrm{H}_{5}$ ) showed antibacterial activity against Escherichia coli, Staphylococcus ayreus and Bacillus subtilis ${ }^{91}$. 
<smiles>[R]c1nn(Cc2n[nH]c(=S)n2-c2ccccc2)c(=O)n1N</smiles><smiles>[R]c1nnn(Cc2nnc(S)n2[R])n1</smiles>

63, $\mathrm{R}=\mathrm{CH}_{3}, \mathrm{CH}_{3} \mathrm{CH}_{2}, \mathrm{CH}_{3} \mathrm{CH}_{2} \mathrm{CH}_{2}$, $\mathrm{C}_{6} \mathrm{H}_{5} \mathrm{CH}_{2}, 4-\mathrm{ClC}_{6} \mathrm{H}_{4} \mathrm{CH}_{2}, \mathrm{C}_{6} \mathrm{H}_{5}$<smiles>[R]n1c(Cn2c(=O)cc(C)[nH]c2=O)n[nH]c1=S</smiles>

64, $\mathrm{R}=\mathrm{C}_{6} \mathrm{H}_{5}, 2-, 3$, and 4- $\mathrm{CH}_{3} \mathrm{C}_{6} \mathrm{H}_{5}$, 4- $\mathrm{ClC}_{6} \mathrm{H}_{4}, 3,4-\mathrm{diCH}_{3} \mathrm{C}_{6} \mathrm{H}_{5}$, $\mathrm{CH}_{3} \mathrm{CH}_{2} \mathrm{OC}_{6} \mathrm{H}_{4}$, $\mathrm{R}^{1}=\mathrm{CH}_{3}, \mathrm{C}_{6} \mathrm{H}_{5}, 4-\mathrm{CH}_{3} \mathrm{C}_{6} \mathrm{H}_{4}$, 4- $\mathrm{ClC}_{6} \mathrm{H}_{4}, 4-\mathrm{Br}-\mathrm{C}_{6} \mathrm{H}_{4}$, $4-\mathrm{FC}_{6} \mathrm{H}_{4}, 4-\mathrm{H}_{3} \mathrm{COC}_{6} \mathrm{H}_{4}$,

65, $\mathrm{R}=\mathrm{C}_{4} \mathrm{H}_{9}, \mathrm{C}_{6} \mathrm{H}_{5} \mathrm{CH}_{2}, \mathrm{C}_{6} \mathrm{H}_{5}$

\section{Figure 11}

The 3-[(2-methyl-1H-3-indolyl)-methyl]-4-aryl-4,5-dihydro-1H-1,2,4-triazole-5-thiones $\mathbf{6 7}^{95}$ and 3-(2-benzothiazolylmethyl)-4-substituted-1,2,4-triazoline-5-thione derivatives $\mathbf{6 8}^{96-99}$

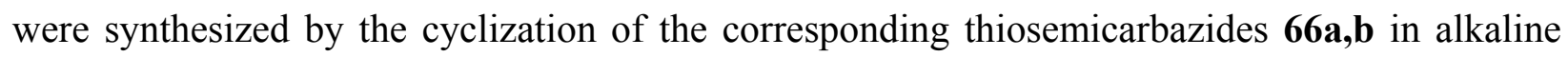
medium (Scheme 21). Compound 65 showed anti-depressant and anti-convulsant activity ${ }^{95}$.

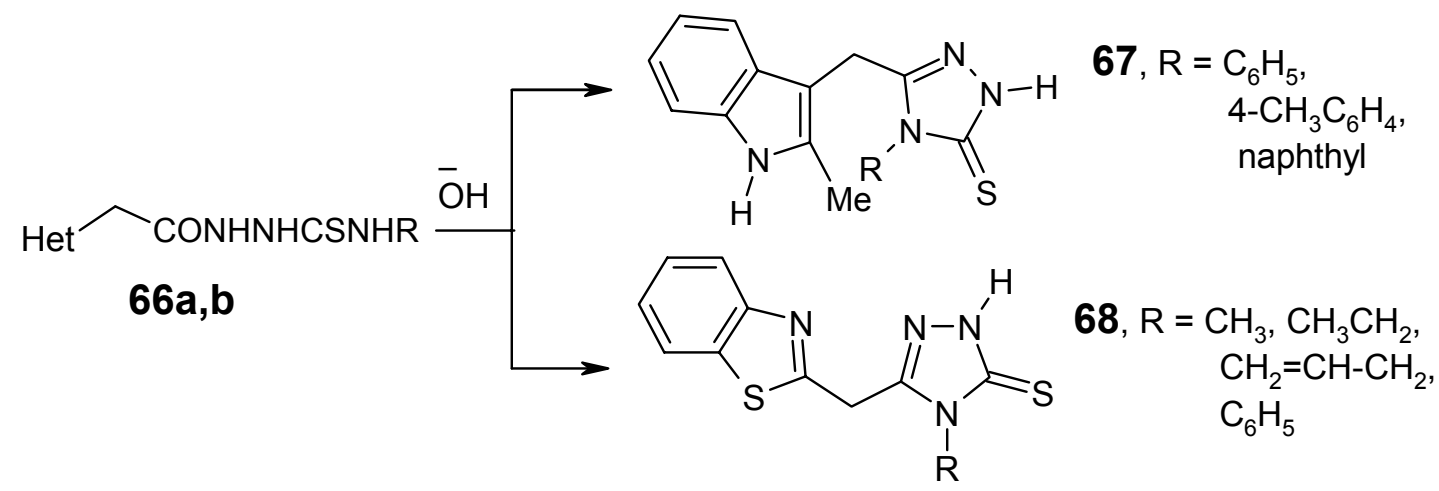

\section{Scheme 21}

A series of 1-(1-carbonylmethyl-1H-benzotriazole)-thiosemicarbazides was synthesized and then cyclized with $\mathrm{NaOH}$ to afford 1-(4-substituted-4H-1,2,4-triazole-3-thion-5-yl)-methyl$1 H$-benzotriazoles $69^{100}$ (Figure 12).<smiles>[R]n1c(S)nnc1Cn1nnc2ccccc21</smiles>

69, $\mathrm{R}=\mathrm{CH}_{3} \mathrm{CH}_{2} \mathrm{CH}_{2} \mathrm{CH}_{2}, \mathrm{C}_{6} \mathrm{H}_{5} \mathrm{CH}_{2}$, $\mathrm{C}_{6} \mathrm{H}_{5}, 4-\mathrm{H}_{3} \mathrm{CC}_{6} \mathrm{H}_{4}, 4-\mathrm{BrC}_{6} \mathrm{H}_{4}$

\section{Figure 12}


The bis(4-aryl)-3-thio-1,2,4-triazol-5-yl)alkanes 71 were prepared by the base cyclization of the corresponding bis(4-arylthiosemicarbazido)alkanes $\mathbf{7 0}^{73,101-103}$ (Scheme 22).

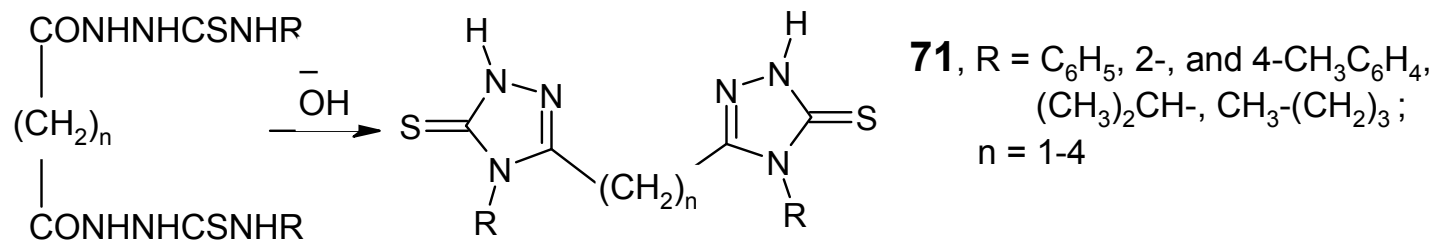

70

\section{Scheme 22}

Shaker et al. ${ }^{104}$ reported that the treatment of 1,4-phenylene-bis-thiosemicarbazide 72 with sodium hydroxide gives 5,5`-(1,4-phenylene)bis(4-aryl-3-mercapto-1,2,4-triazole) 73 (Scheme 23).
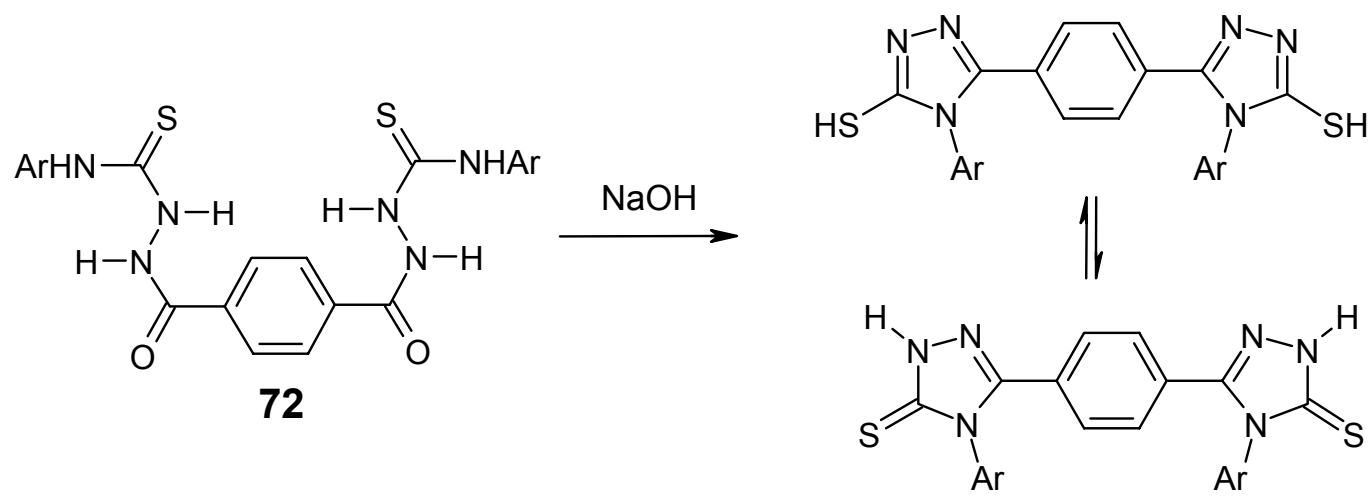

73, $\mathrm{Ar}=\mathrm{C}_{6} \mathrm{H}_{5}, \mathrm{C}_{6} \mathrm{H}_{5} \mathrm{CH}_{2}$

\section{Scheme 23}

A series of hetero-substituted thiosemicarbazides was synthesized and then cyclized in alkaline medium to afford the corresponding triazolethiones $\mathbf{7 4}^{43}, \mathbf{7 5}^{\mathbf{7 7 , 1 0 5}}, \mathbf{7 6}^{69,106}, \mathbf{7 7}^{107,108}$, and $\mathbf{7 8}^{109}$ in excellent yields (Figure 13). Compound 77 exhibited potential and broad-spectrum antitumor activity against most of the tested subpanel tumour cell lines (GI50, TGI and LC50 values $<100 \mu \mathrm{M})^{107}$. Compound 78 exhibited moderate inhibitory activity against plant pathogenic fungi such as cucumber grey mold, rape scherotium, wheat gibberella and cotton damping-off at $50 \mu \mathrm{g} / \mathrm{mL}$ concentration ${ }^{109}$. 
<smiles>[R]N1C(=O)CCC1c1n[nH][nH]c1=S</smiles>

74, $\mathrm{R}=\mathrm{H}, \mathrm{C}_{6} \mathrm{H}_{5} \mathrm{CH}_{2}$; $\mathrm{R}^{1}=\mathrm{H}_{3} \mathrm{C}, \mathrm{C}_{6} \mathrm{H}_{5}$,<smiles>[R1]c1cn([Al])nc1C1=NNNC(=S)N1[R]</smiles>

77, $\mathrm{R}=$ cyclohexyl, $\mathrm{C}_{6} \mathrm{H}_{5}, 4-\mathrm{CH}_{3} \mathrm{OC}_{6} \mathrm{H}_{4}$, 4- $\mathrm{CH}_{3} \mathrm{C}_{6} \mathrm{H}_{4}, 4-\mathrm{ClC}_{6} \mathrm{H}_{4}, 4-\mathrm{FC}_{6} \mathrm{H}_{5}$, $\mathrm{R}^{1}=\mathrm{OH} . \mathrm{OCH} 3$, $\mathrm{Ar}=\mathrm{C}_{6} \mathrm{H}_{5}, 4-\mathrm{ClC}_{6} \mathrm{H}_{4}$<smiles>[R1]c1ccc(-c2nnc(S)n2[R])o1</smiles><smiles>[R]n1c(-c2ncoc2C)n[nH]c1=S</smiles>

75, $\mathrm{R}=\mathrm{CH}_{3}, \mathrm{C}_{4} \mathrm{H}_{9}$, cyclohexyl, $\mathrm{C}_{6} \mathrm{H}_{5}$, 4- $\mathrm{CH}_{3} \mathrm{C}_{6} \mathrm{H}_{4}, 4-\mathrm{FC}_{6} \mathrm{H}_{4}, 4-\mathrm{ClC}_{6} \mathrm{H}_{4}$, 4- $\mathrm{BrC}_{6} \mathrm{H}_{4}, 4-\mathrm{O}_{2} \mathrm{NC}_{6} \mathrm{H}_{4}$; $\mathrm{R}^{1}=\mathrm{H}, \mathrm{NO}_{2}$<smiles>Cc1c(-c2n[nH][nH]c2=S)nnn1-c1ccccc1</smiles>

78, $\mathrm{Ar}=\mathrm{C}_{6} \mathrm{H}_{5}, 2-$, and 4- $\mathrm{CH}_{3} \mathrm{C}_{6} \mathrm{H}_{4}$,

2-, and $4-\mathrm{CH}_{3} \mathrm{OC}_{6} \mathrm{H}_{4}$,

3-, and $4-\mathrm{ClC}_{6} \mathrm{H}_{4}$, 2-, 3-, and 4- $\mathrm{BrC}_{6} \mathrm{H}_{4}$, $3-\mathrm{CF}_{3} \mathrm{C}_{6} \mathrm{H}_{4}, 2,4-\mathrm{Cl}_{2} \mathrm{C}_{6} \mathrm{H}_{3}$

\section{Figure 13}

The isomeric substituted thiosemicarbazides 79, when subjected to react with $4 N \mathrm{NaOH}$, underwent intramolecular dehydrate cyclization to furnish the corresponding 4-alkyl/aryl-5(isomeric pyridoyl)-1,2,4-triazole-3-thioles $\mathbf{8 0}^{54,110-113}$ (Scheme 24). Compound 80 (3-pyridyl) exhibit moderate inhibitory activities at $32 \mu \mathrm{g} / \mathrm{mL}$ against $S$. aureus $^{113}$.<smiles>[R]NC(=S)N(NO)NC(=O)c1cccnc1</smiles><smiles>[R]n1c(S)nnc1-c1cccnc1</smiles>

80, $\mathrm{R}=\mathrm{C}_{2} \mathrm{H}_{5}, \mathrm{C}_{6} \mathrm{H}_{5}, \mathrm{C}_{6} \mathrm{H}_{4} \mathrm{CH}_{2} \mathrm{CH}_{2}, 4-\mathrm{CH}_{3} \mathrm{C}_{6} \mathrm{H}_{4}$, naphthyl

\section{Scheme 24}

Also, 1,4-disubstituted-thiosemicarbazides $\mathbf{8 1}$ were synthesized from the corresponding carbohydrazides and cyclized under mildly basic conditions to $1,2,4$-triazoles $\mathbf{8 2}{ }^{114}, \mathbf{8 3}^{115}, \mathbf{8 4}^{116}$ and $\mathbf{8 5}^{117}$ (Figure 14). Compound $84\left(\mathrm{R}=2-\mathrm{Cl}, \mathrm{Ar}=3-\mathrm{FC}_{6} \mathrm{H}_{4}\right)$ exhibited good anti-fungal activity $^{116}$. 


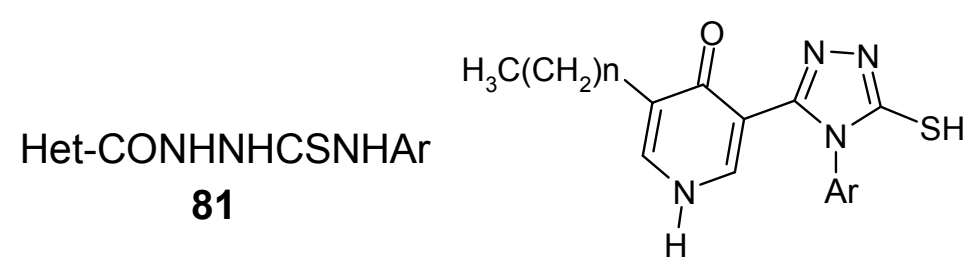

82, $\mathrm{Ar}=\mathrm{CH}_{3}, \mathrm{C}_{6} \mathrm{H}_{5}$; $\mathrm{n}=3,4,6$<smiles>[R]c1ccccc1-n1nc(-c2nnc(S)n2[Al])c(=O)cc1C</smiles>

84, $\mathrm{R}=2-$, and 4-Cl; $\mathrm{Ar}=2-$, and $3-\mathrm{FC}_{6} \mathrm{H}_{4}$<smiles>[R]c1nc([R1])c(-c2n[nH]c(=S)n2[Al])nc1[R]</smiles>

85, $\mathrm{Ar}=\mathrm{CH}_{3} \mathrm{CH}_{2}, \mathrm{C}_{6} \mathrm{H}_{5}$,

$\mathrm{R}^{1}=\mathrm{H}, \mathrm{NH}_{2}$;

$\mathrm{R}^{2}=\mathrm{H}, \mathrm{Cl}$;

$\mathrm{R}^{3}=\mathrm{H}, \mathrm{NH}_{2}$,

\section{Figure 14}

The 5-(2-phenyl-benzimidazol-1-yl-methyl)-4-aryl-4H-1,2,4-triazole-3-thiones were synthesized, and their in vitro effects on the rat liver microsomal NADPH-dependent lipid per oxidation (LP) levels were determined ${ }^{118}$. Also, the triazoles $\mathbf{8 6}^{119}, \mathbf{8 7}^{52}, \mathbf{8 8}^{120}$ and $\mathbf{8 9}{ }^{121-124}$ were synthesized by the cyclization of the corresponding thiosemicarbazides in alkaline medium (Figure 15).<smiles>S=c1[nH]nc(-c2cn(Cc3ccccc3)c3ccccc23)n1-c1ccccc1</smiles>

86<smiles>CN1C(=O)C(c2nnc(S)n2-c2ccccc2)N=C(c2ccccc2)c2cc(Cl)ccc21</smiles>

88<smiles></smiles>

87, $\mathrm{R}=\mathrm{CH}_{3}, \mathrm{CH}_{3} \mathrm{CH}_{2}, \mathrm{CH}_{2}=\mathrm{CH}-\mathrm{CH}_{2}, \mathrm{C}_{6} \mathrm{H}_{5}$<smiles>[R]n1c(-c2nn(-c3ccccc3Cl)c3nc4ccccc4nc23)n[nH]c1=S</smiles>

89, $\mathrm{R}=$ methyl, allyl

Figure 15 


\subsection{Synthesis of 2,4-dialkyl/aryl-1,2,4-triazolethiones}

The oxidative cyclization of aldehyde thiosemicarbazones $\mathbf{9 0}$ with ferric chloride solutions gave 1,2,4-triazoline 91 and 1,3,4-thiadiazoline $\mathbf{9 2}^{125-127}$ (Scheme 25).

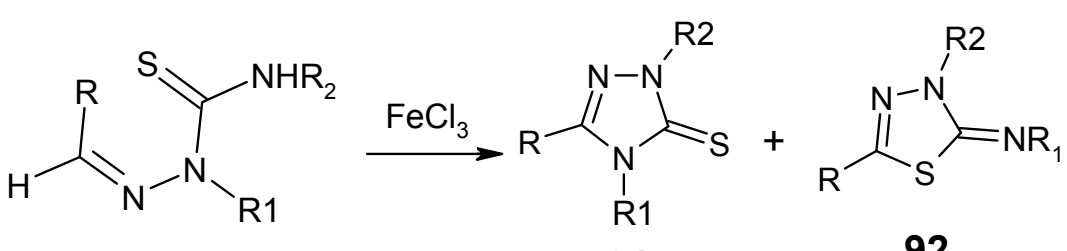

90

91

92

$$
\begin{aligned}
& \mathrm{R}=\mathrm{R}^{1}=\mathrm{CH}_{3}, \mathrm{CO}_{2} \mathrm{CH}_{2} \mathrm{CH}_{3}, \mathrm{C}_{6} \mathrm{H}_{5}, \mathrm{C}_{6} \mathrm{H}_{5} \mathrm{CO}, 3-\text {, and 4- } \mathrm{CH}_{3} \mathrm{C}_{6} \mathrm{H}_{4}, 3-\text {, and 4- } \mathrm{ClC}_{6} \mathrm{H}_{4}, 4-\mathrm{F}_{3} \mathrm{CC}_{6} \mathrm{H}_{4}, \\
& \quad 3-, \text { and 4- } \mathrm{BrC}_{6} \mathrm{H}_{4}, 4-\mathrm{H}_{3} \mathrm{COC}_{6} \mathrm{H}_{4}, 4-\left(\mathrm{H}_{3} \mathrm{C}\right)_{2} \mathrm{NC}_{6} \mathrm{H}_{4}, 4-\mathrm{O}_{2} \mathrm{NC}_{6} \mathrm{H}_{4} ; \\
& \mathrm{R}^{2}=\mathrm{CH}_{3}, \mathrm{C}_{6} \mathrm{H}_{5},
\end{aligned}
$$

\section{Scheme 25}

The photochemistry behavior of some substituted aldehyde thiosemicarbazones $\mathbf{9 3}$ have been investigated in methanol at $254 \mathrm{~nm}$ and cyclized to furnish the 5-thioxo-1,2,4-triazolines $\mathbf{9 5}^{128,129}$ (Scheme 26). The first step of the photoreaction of compound 93 depicted as the cyclization to the 1,2,4-triazolidinethiones 94, the second step as the photo oxidation of 94 to give $95^{129}$.<smiles>[R]NC(=S)N([R1])N=C([R])C</smiles>

93<smiles>[R]C1N([R])C(=S)N([R7])N1[R]</smiles>

94<smiles>CCC</smiles><smiles>[R]c1nn([R7])c(=S)n1[R]</smiles>

95, $\mathrm{R}=\mathrm{CH}_{3}, \mathrm{C}_{6} \mathrm{H}_{5}, \mathrm{C}_{6} \mathrm{H}_{5} \mathrm{CH}_{2}$, $\mathrm{R}^{1}, \mathrm{R}^{2}=\mathrm{CH}_{3}, \mathrm{C}_{6} \mathrm{H}_{5}$

\section{Scheme 26}

The reactions between 2,4-disubstituted thiosemicarbazides and orthoesters in refluxing xylene led to the formation of the 1,2,4-triazoline-5-thiones $\mathbf{9 6}^{130}$ (Figure 16). 
<smiles>[R]c1nn(C)c(=S)n1[R]</smiles>

96, $\mathrm{R}=\mathrm{CH}_{3}, \mathrm{CH}_{3} \mathrm{CH}_{2}, \mathrm{C}_{6} \mathrm{H}_{5}$; $\mathrm{R}^{1}=\mathrm{H}_{3} \mathrm{C}, \mathrm{C}_{6} \mathrm{H}_{5}$,<smiles>[R]c1cc(-n2c(C)nn([R])c2=S)n([R])n1</smiles>

97, R, $\mathrm{R}^{1}, \mathrm{R}^{2}=\mathrm{H}_{3} \mathrm{C}, \mathrm{C}_{6} \mathrm{H}_{5}$,

\section{Figure 16}

Also, cyclization of pyrazolyl thiosemicarbazides with formic acid-acetic anhydride or with triethyl orthoacetate-acetic anhydride provided 5-methyl-4-(pyrazol-5-yl)-1,2,4-triazole-3thiones $97^{131}$ (Figure 16).

5-Aryl-2,4-dialkyl-2,4-dihydro-3H-1,2,4-triazol-3-ones were converted in 55-74\% yield to the corresponding $3 H-1,2,4$-triazole-3-thiones by using the combination bis(tricyclohexylstannyl) sulfide/boron-trichloride ${ }^{132}$.

The 2,4-dimethyl-5-(2-fluorophenyl)-5-phenyl-1,2,4-triazolidine-3-thione (100) ${ }^{61}$ was prepared in low yield by heating a methanolic solution of 2-fluorobenzophenone (98) and 2,4dimethyl-thiosemicarbazide (99) in the presence of $\mathrm{KOH}$ (Scheme 27).

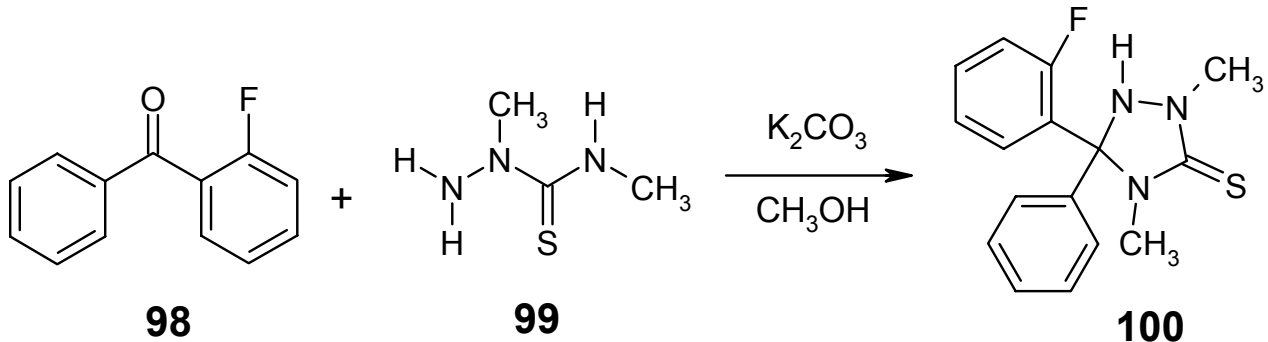

\section{Scheme 27}

The carbo(3-phenylpyrazol-5-yl-hydrazonoyl)halides $\mathbf{1 0 1}$ reacted with phenyl isothiocyanate to yield 4-phenyl-1-(3-phenylpyrazol-5-yl)-3-substituted- $\Delta^{2}-1,2,4$-triazoline-5thiones $\mathbf{1 0 2}^{133}$ (Scheme 28). 
<smiles></smiles>

\section{Scheme 28}

1,2,4-Triazole-3-thiones $\mathbf{1 0 3}^{39}, \mathbf{1 0 4}^{134}$ and $\mathbf{1 0 5}^{118}$ were obtained from the corresponding thiosemicarbazones in alkaline medium (Figure 17).<smiles>CC(=O)OCCn1c(-c2ccccc2)nn(C(C)=O)c1=S</smiles><smiles>[R3]c1nc([R1])c(-c2nn(C)c(=S)n2[R])nc1[R3]</smiles><smiles>C=C(C)Cn1c(NC(C)=O)nn(C)c1=S</smiles>

104, $\mathrm{R}=\mathrm{CH}_{3} \mathrm{CH}_{2} \mathrm{O}, \mathrm{C}_{6} \mathrm{H}_{5}$

105, $\mathrm{R}=\mathrm{CH}_{3} \mathrm{CH}_{2}, \mathrm{C}_{6} \mathrm{H}_{5}$;

$\mathrm{R}^{1}=\mathrm{R}^{2}=\mathrm{H}, \mathrm{NH}_{2}$, $\mathrm{R}^{3}=\mathrm{H}, \mathrm{Cl}$,

\section{Figure 17}

The products of the condensation of 5-hydroxy-3,3,5-trimethylisoxazolidine (106) with 4phenyl-/2-methyl-4-phenylthiosemicarbazide have predominantly 1,2,4-triazolidine or isoxazolidine structure and do not display ring-ring tautomeric interconversion in solution ${ }^{135}$.

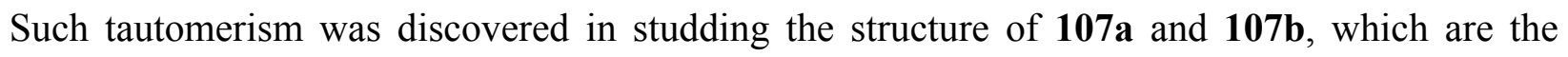
products of 106 with 4-methyl- and 2,4-dimethylthiosemicarbazides. Thiones 107a and 107b were formed after brief heating of the starting reagents in methanol at reflux in the presence of catalytic amount of acetic acid $^{136}$ (Scheme 29). 


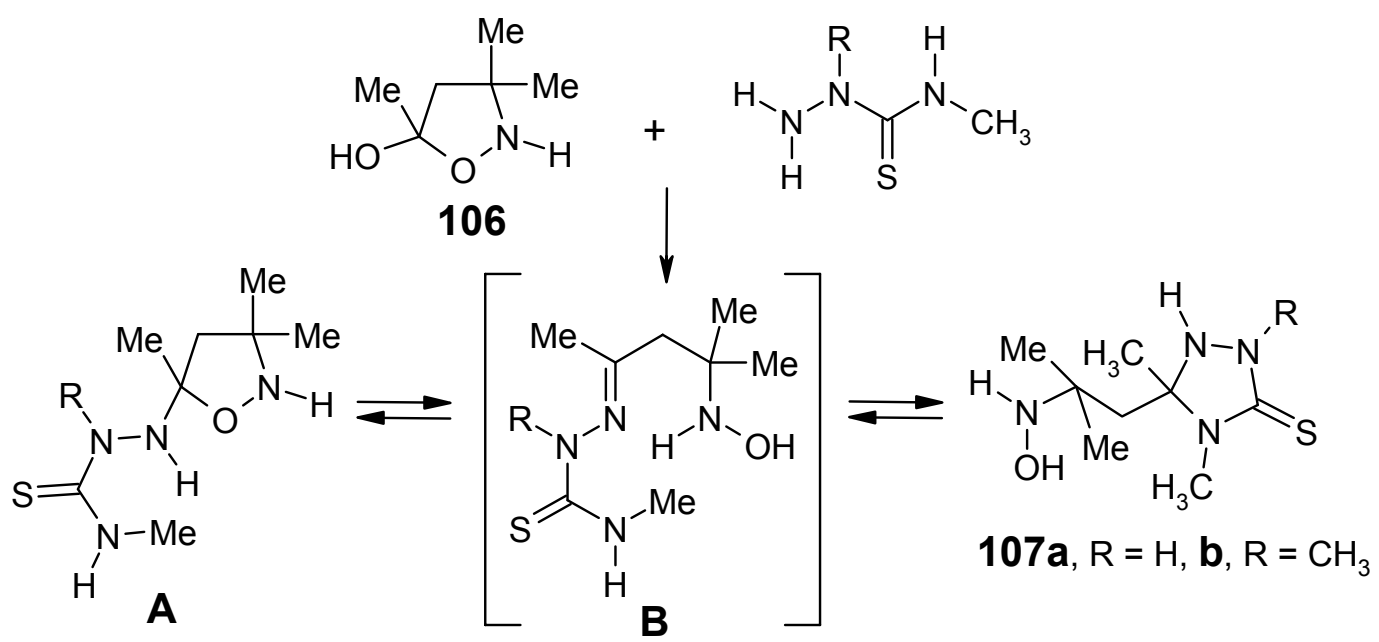

\section{Scheme 29}

The oxidation of 2-methyl-4-phenyl thiosemicarbazides 108 with cupric perchlorate in methanol gave 1,2,4-triazolines $\mathbf{1 0 9}^{129}$ (Scheme 30).

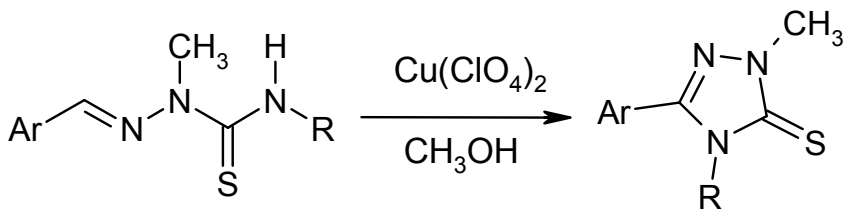

108
109, $\mathrm{Ar}=\mathrm{C}_{6} \mathrm{H}_{5}, p-\mathrm{CH}_{3} \mathrm{C}_{6} \mathrm{H}_{4}$, 4- $\mathrm{ClC}_{6} \mathrm{H}_{4}, 4-\mathrm{BrC}_{6} \mathrm{H}_{4}$, 4- $\mathrm{CH}_{3} \mathrm{OC}_{6} \mathrm{H}_{4}, 4-\mathrm{CF}_{3} \mathrm{C}_{6} \mathrm{H}_{4}$ $\mathrm{R}=\mathrm{C}_{6} \mathrm{H}_{5}, 4-\mathrm{CH}_{3} \mathrm{C}_{6} \mathrm{H}_{4}$, $4-\mathrm{ClC}_{6} \mathrm{H}_{4}, 4-\mathrm{CH}_{3} \mathrm{OC}_{6} \mathrm{H}_{4}$

\section{Scheme 30}

\subsection{Synthesis of 1,2,4-triazole-3,5-dithiones}

Alkali-catalyzed thermal cyclization of 1-alkyl- and 1,8-dialkyl-2,5-dithiobiureas 110 ( $\mathrm{R}=$ alkyl, $\mathrm{R}^{1}=\mathrm{H} ; \mathrm{R}, \mathrm{R}^{1}=$ alkyl) forms 4-alkyl-1,2,4-triazolidine-3,5-dithiones $\mathbf{1 0 8}(\mathrm{R}=\mathrm{Me}, \mathrm{Et})$ and 1,3,4thiadiazoline $112(\mathrm{R}=\mathrm{Pr}, \mathrm{Bu})$. Under the same conditions, 1-alkyl-6-aryl-2,5-dithiobiureas give $112(\mathrm{R}=\mathrm{Ph}$, substituted $\mathrm{Ph})$ and 111 when the alkyl groups are methyl or ethyl ${ }^{137}$ (Scheme 31$)$. 


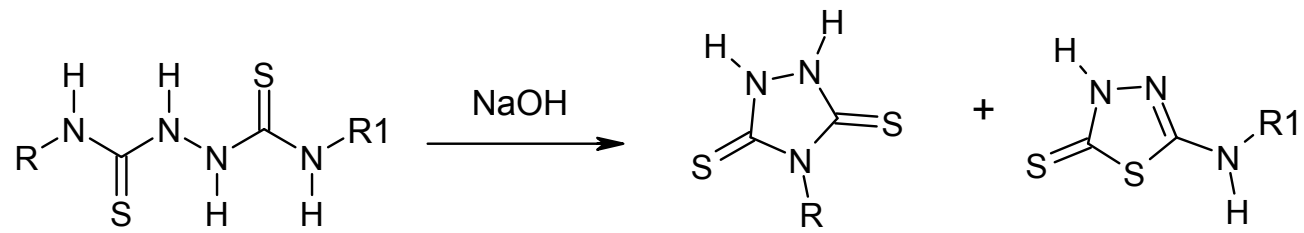

$$
\begin{aligned}
& 110 \\
& 111(30-34 \%) \quad 112(40-44 \%) \\
& \mathrm{R}=\mathrm{CH}_{3}, \mathrm{CH}_{3} \mathrm{CH}_{2} ; \mathrm{R}^{1}=\mathrm{H} \text {, alkyl, } \mathrm{C}_{6} \mathrm{H}_{5} 4-\mathrm{CH}_{3} \mathrm{OC}_{6} \mathrm{H}_{4}, 4-\mathrm{CH}_{3} \mathrm{CH}_{2} \mathrm{OC}_{6} \mathrm{H}_{4}, 4-\mathrm{ClC}_{6} \mathrm{H}_{4}
\end{aligned}
$$

\section{Scheme 31}

\subsection{Synthesis of fused mercapto/thione-1,2,4-triazole heterocycles}

Shaker ${ }^{138}$ reported that the addition-condensation of thiosemicarbazones 113 to mercaptoacetic acid furnished the corresponding cycloalkane spirothiazolidin-4-ones 114, which on treatment with $\mathrm{NaOH}$ underwent cyclization to spiro[1,3]thiazolo[3,4-b]-1,2,4-triazoles 115 (Scheme 32).

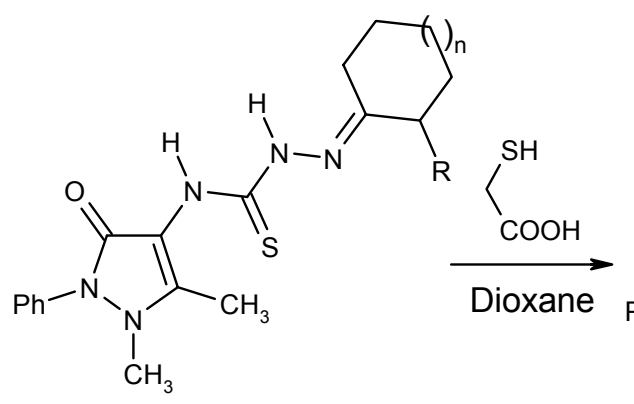

113<smiles>[R]C1CCCCC12SCC(=O)N2NC(=S)Nc1c(C)n(C)n(-c2ccccc2)c1=O</smiles>

114

$\mathrm{NaOH} / \mathrm{MeOH}$

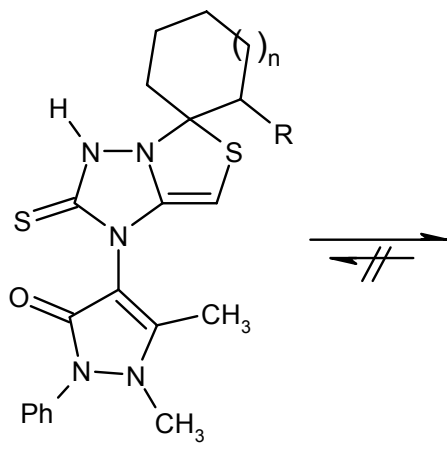<smiles>[R]CC12CCCCC1C21SC=C2N(c3c(C)n(C)n(-c4ccccc4)c3=O)C(S)=NN21</smiles>

115, $\mathrm{R}=\mathrm{H}, \mathrm{CH}_{3}, \mathrm{n}=1,2$

\section{Scheme 32}


The 1,2,4-triazolo[4,3-c]pyrimidines $\mathbf{1 1 6}^{139}, \quad$ 1,2,4-triazolo[4,5-b]pyrazin-2(1H)-ones $\mathbf{1 1 7 ^ { 1 4 0 }}$ and 1,2,4-triazolo[3,4-f][1,2,4]triazinone $\mathbf{1 1 8}^{141}$ were synthesized from the reaction of the corresponding hydrazine derivatives with carbon disulfide (Figure 18). Moreover, 1,2,4triazolo[4,3-d][1,2,4]triazine 116 was prepared by nucleophilic cleavage of furan ring of [1]benzofuro[2,3-e][1,2,4] triazines $^{142}$ (Figure 18).<smiles>[R]c1nc(-c2ccccc2)c(C#N)c2n[nH]c(=S)n12</smiles>

116, $\mathrm{R}=\mathrm{C}_{6} \mathrm{H}_{5}$,<smiles>[R]n1cc(Cl)n2c(S)nnc2c1=O</smiles>

117, $\mathrm{R}=\mathrm{CH}_{3}, \mathrm{C}_{6} \mathrm{H}_{5}$<smiles>Nc1nc(=O)c2n[nH]c(=S)n2[nH]1</smiles>

118 $\mathrm{C}_{6} \mathrm{H}_{5} \mathrm{CH}_{2} \mathrm{NH}$,<smiles>O=c1n(-c2ccccc2)nc(-c2ccccc2O)c2n[nH]c(=S)n12</smiles>

119

\section{Figure 18}

When compound $\mathbf{1 2 0}$ was allowed to react with $\mathrm{CS}_{2}$, the pyrazolotriazolo-pyrimidine-2thione 121 was obtained ${ }^{143}$ (Scheme 33).<smiles>N=c1c2cnn(-c3ccccc3)c2ncn1N</smiles>

120

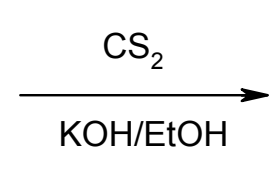

$122^{144}$ thienopyrimidotriazoles $\mathbf{1 2 3}^{145}$ and triazolopyrimidopyridazine $\mathbf{1 2 4}^{146}$ were prepared from the reaction of $\mathrm{CS}_{2}$ with the corresponding hydrazine derivatives (Figure 19). 
<smiles>Cc1nn2c(=S)[nH]nc2c2ccccc12</smiles>

122<smiles></smiles>

123, $\mathrm{R}=\mathrm{CH}_{3} \mathrm{CH}_{2} \mathrm{O}, \mathrm{CH}_{3}, \mathrm{NH}_{2}$<smiles>N=C1c2c(nnc(-c3ccccc3)c2-c2ccccc2)-n2c1n[nH]c2=S</smiles>

124

\section{Figure 19}

The tricyclic 3(2H)-thioxo-1,2,4-triazolo[4,3-b]pyridazine-6(5H)-ones $\mathbf{1 2 5}$ were synthesized from the reaction of phenyl isothiocyanate with the corresponding hydrazine derivatives $^{147}$ (Figure 20).<smiles>[Y20]C1c2n[nH]c(=S)n2NC(=O)C1[Y]=[V]</smiles>
a, $X=Y=W=Z=C H$
b, $X=N, Y=W=Z=C H$
c, $Y=N, X=W=Z=C H$
$\mathrm{d}, \mathrm{W}=\mathrm{N}, \mathrm{X}=\mathrm{Y}=\mathrm{Z}=\mathrm{CH}$
e, $Z=N, X=Y=W=C H$

\section{Figure 20}

A rearrangement reaction about 2-benzothiazolylthioacetyl hydrazide (126) to produce $s$ triazolo[3,4-b]benzothiazol-3-thiol (128) in the presence of $\mathrm{KOH}$ and $\mathrm{CS}_{2}$ was described. Other way to synthesis $\mathbf{1 2 8}$ from 2-benzothiazolylhydrazine (127) under the same conditions ${ }^{148,149}$ (Scheme 34).<smiles>O=C(CSc1nc2ccccc2s1)NNNC(=O)C(O)C(O)C(=O)NNc1nc2ccccc2s1</smiles>

\section{7}

\section{Scheme 34}


8,9-Di(2-furyl)-2,3-dihydro-5-methylfuro[3,2-e][1,2,4]triazolo[1,5-c]pyrimidine-2-thione (130) was prepared from the reaction of compound 129 reacted with carbon disulfide ${ }^{150}$ (Scheme $35)$.<smiles>Cc1nc2oc(-c3ccco3)c(-c3ccco3)c2c(=N)n1N</smiles>

129

130

\section{Scheme 35}

A series of 1,2,4-triazolo[2,3-d][1,5]benzoxazepin-2-thiones 132 were achieved via acidinduced ring closure of the geminal arylazo-isothiocyanate compounds $\mathbf{1 3 1}^{151}$ (Scheme 36).

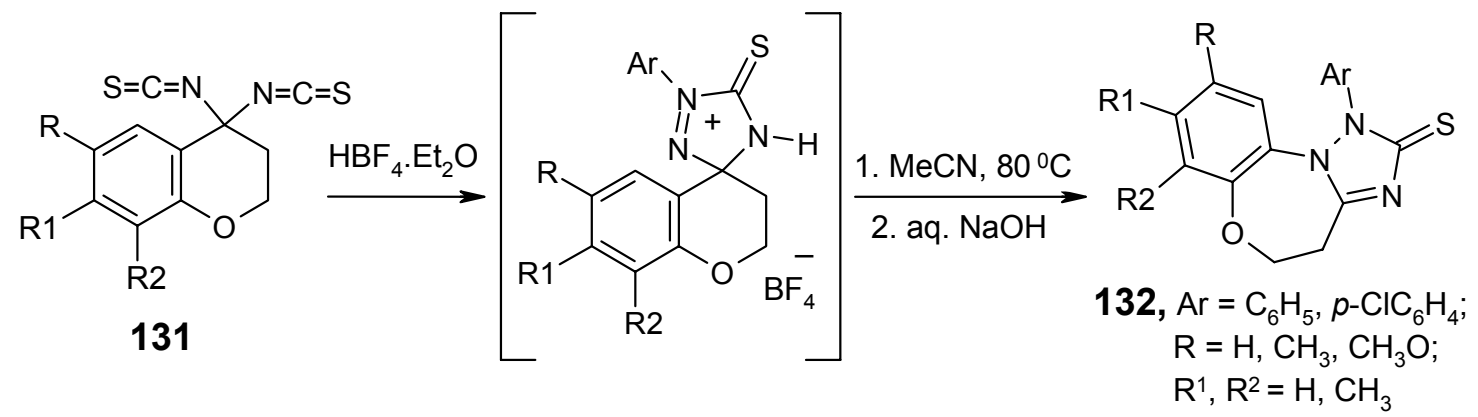

\section{Scheme 36}

The cyclization of the 4-amino-3-thioxotriazolylindazole 133 gave the 3-thioxo-triazole $\mathbf{1 3 4}^{98}$, while the triazole 135 was synthesized from pyrrolo[1,2-a]thieno[2,3-e]-pyrazin-5-one ${ }^{152}$ (Figure 21).<smiles>Nn1c(-c2n[nH]c3ccccc23)n[nH]c1=S</smiles>

133<smiles></smiles>

134<smiles>S=c1[nH]nc2c3cccn3c3ccsc3n12</smiles>

Figure 21 
The benzofuro[2,3- $d$ ] pyridazine fused with 1,2,4-triazole 136 was prepared by the ring closure of 4-hydrazino[1]benzofuro[2,3- $d$ ]pyridazine, derived from naturally occurring rotenone $^{153}$ (Figure 22).

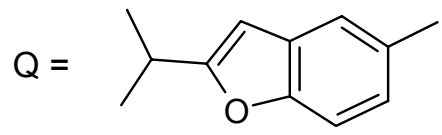<smiles>COc1cc2oc3c(c(O)nn4c(=S)[nH]nc34)c2cc1OC</smiles>

136

\section{Figure 22}

The treatment of 4-hydrazino-pyrano[3`,4:5,6]pyrido[2,3-d]pyrimidine 137 with carbon disulfide led to the formation of pyrano[5 $\left., 4^{`}: 5,6\right]$ pyrido[3,2-e]triazolo[4,3-c]-pyrimidine $\mathbf{1 3 8}^{154}$ (Scheme 37).<smiles></smiles>

\section{Scheme 37}

Refluxing 3-hydrazino[1,2,4]triazino[5,6-b]indole 139 with carbon disulfide produced 1,2dihydro-10H-[1,2,4]triazolo[3 $\left., 44^{`}: 3,4\right][1,2,4]$ triazino[5,6-b]indole-1-thione $\mathbf{1 4 0}^{155}$ (Scheme 38).<smiles>NNc1nnc2c(n1)[nH]c1ccccc12</smiles>

139<smiles>S=c1[nH]nc2nnc3c(n12)NNc1ccccc1-3</smiles>

140

\section{Scheme 38}

Similarly, 1,2,4-triazolo[4',3':4,5][1,2,4]triazino[3,2-b]quinazolin-7-one 141 was prepared from the corresponding hydrazine ${ }^{156}$ (Figure 23). 


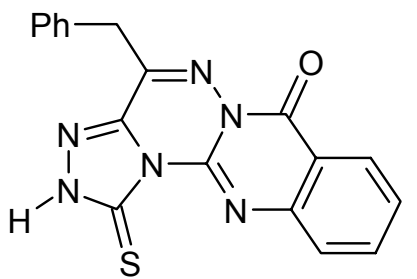

141

Figure 23

When the compound 142 was allowed to react with carbon disulfide, the 3-mercapto-10methyl-8-phenyl-1(1H)-triazolo[3“",4” $: 2,3]$ pyrimido[ $\left[5^{\prime}, 6^{\prime}: 4,5\right]$ thieno[2,3-d]pyrimidin-5-one (143) was obtained ${ }^{157}$ (Scheme 39).<smiles>Cc1nc(-c2ccccc2)nc2sc3c(=O)n(CC(C)(F)F)c(N)nc3c12</smiles>

142<smiles>Cc1nc(-c2ccccc2)nc2sc3c(=O)n4c(S)n[nH]c4nc3c12</smiles>

143

\section{Scheme 39}

Also, the treatment of $\mathbf{1 4 4}$ with carbon disulfide in alcoholic potassium hydroxide solution gave benzopyrano[3 $\left.3^{`}: 5,6\right]$ pyrano[3,2-e][1,2,4]triazolo[1,5-c]pyrimidine-2-thione $\quad \mathbf{1 4 5}^{158}$ (Scheme 40).<smiles>CC(C)(C)C(C)(C)C</smiles>

144<smiles>O=c1oc2ccccc2c2c1C(c1ccc(Cl)cc1)c1c(ncn3[nH]c(=S)nc13)O2</smiles>

145

\section{Scheme 40}




\section{Chemical reactivity}

\subsection{Alkylation and arylation}

The reaction of 1,2,4-triazoline-5-thione 6 with acrylonitrile afforded the $N$-substituted adduct $\mathbf{1 4 6}^{159}$. Triazoles 147 were prepared as potential fungicides by treating 3 with $\mathrm{RCOCH}_{2} \mathrm{R}^{2}\left(\mathrm{R}^{2}=\right.$ $\mathrm{Cl}, \mathrm{Br})^{160}$ (Figure 24).<smiles>N#CCCn1c(-c2ccccc2)n[nH]c1=S</smiles>

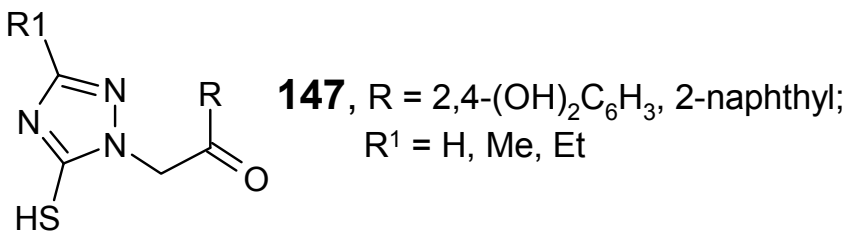

146

\section{Figure 24}

Catalytic vinylation of triazolethione 6 by acetylene over $\mathrm{Cd}(\mathrm{OAc})_{2}$ or $\mathrm{CuCl}$ at $15 \mathrm{~atm}$. gave mixtures containing triazoles 148 and $149^{161}$ (Figure 25).

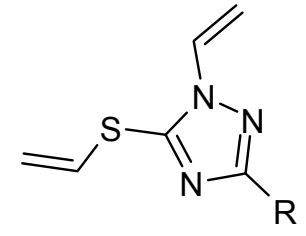

148<smiles>[R]c1nc(SC=C)nn1C=C</smiles>

149

\section{Figure 25}

The triazole $\mathbf{1 5 0}$ was prepared in 55-88.2\% yield by treatment of 3 with $\mathrm{R}^{1} \mathrm{C}$.tplbond.CCOPh. The same reaction in the presence of $2 \% \mathrm{NaOH}$ gave a mixture of $\mathbf{1 5 1}$ and $\mathbf{1 5 2}^{162}$. Also, reaction of 3 with PhC.tplbond.CCN in a molar ratio (1:2) gave $\mathbf{1 5 3}^{163}$ (Figure 26). 


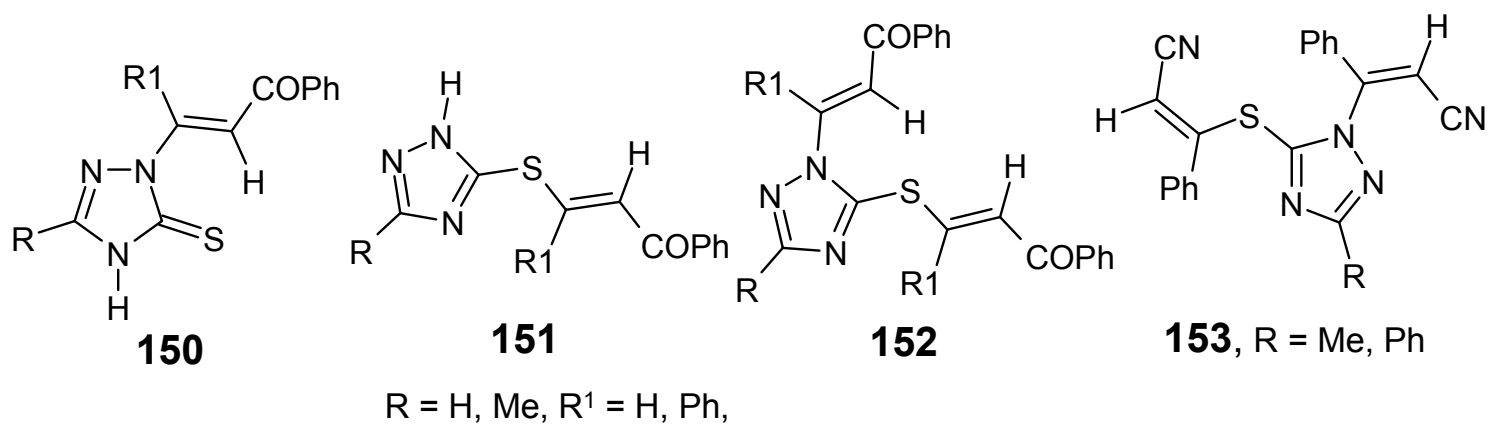

Figure 26

The alkylation of 3 with alkyl halide in refluxing ethanol gave 32-99\% yields of 5alkylthio-1,2,4-triazoles $\mathbf{1 5 4}{ }^{164,165}$ which had moderate bacteriostatic activity and diuretic activity that increased with the size of R (Figure 27).

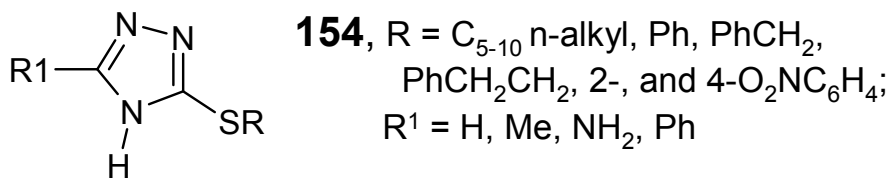

\section{Figure 27}

The $S$-alkylated derivatives $\mathbf{1 5 5}$ were prepared by the alkylation of the corresponding triazolethiones ${ }^{23,64,166}$ (Figure 28).

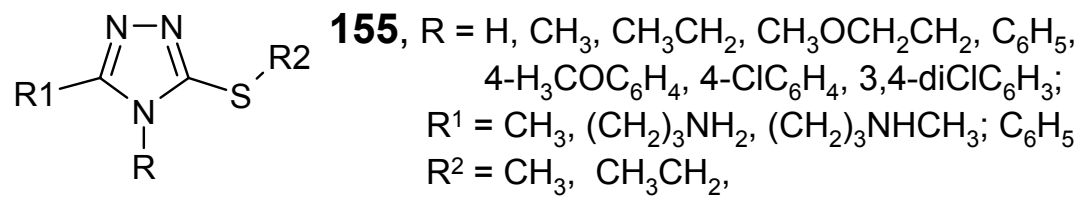

\section{Figure 28}

3-Vinylthio-1,2,4-triazoles 156 were prepared in 35-80\% yields by addition of HC.tplbond.CH to the corresponding triazolethione in the presence of $\mathrm{KOH}$ in an autoclave $2 \mathrm{~h}$ at $14 \mathrm{~atm}$. and $160{ }^{\circ} \mathrm{C} .{ }^{167}$ The reaction of triazole 3 with ethyl bromoacetate or propynyl bromide leads to the ethyl-(3-substituted-1,2,4-triazol-5-yl-thio)acetate $157^{168}$ and prop-3-ynylthio- $S$ triazoles $\mathbf{1 5 8}^{169,170}$ (Figure 29). 


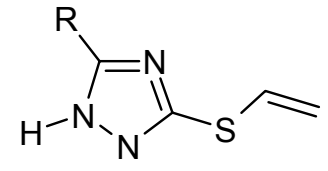<smiles>[R]c1n[nH]c(SCC(=O)OCC)n1</smiles><smiles>[R]c1n[nH]c(SCC#C)n1</smiles>

156, $\mathrm{R}=\mathrm{H}, \mathrm{Me}, 2$-furyl

157, $\mathrm{R}=\mathrm{H}, \mathrm{CH}_{3}, \mathrm{C}_{2} \mathrm{H}_{5}$

158, $R=$ alkyl, aryl

\section{Figure 29}

Addition reaction of epichlorohydrin with the corresponding triazolethiones gave $S$ alkylated product $\mathbf{1 5 9} 9^{171,172}$ (Figure 30).

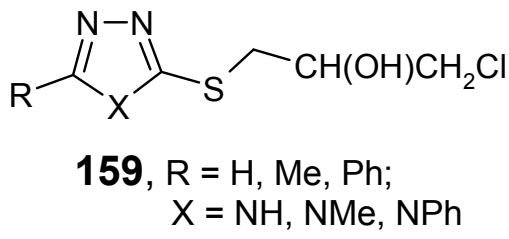

\section{Figure 30}

When 1-(2-chloroethyl)-4-(2-methoxyphenyl)piperazine reacted with triazoles 6 in alkaline medium gave both the $S$-alkylated 160 and the $N$-alkylated isomers 161 were obtained ${ }^{69,173}$ (Figure 31).<smiles>[X]c1ccccc1N1CCN(CCSc2nnc([Al])n2[R])CC1</smiles><smiles>[X]c1ccccc1N1CCN(CCn2nc([Al])n([R])c2=S)CC1</smiles>

$$
\mathrm{R}=\mathrm{H}, \mathrm{NH}_{2} ; \mathrm{Ar}=\mathrm{C}_{6} \mathrm{H}_{5}, 2-\text {, and 4- } \mathrm{ClC}_{6} \mathrm{H}_{4}, 4-\mathrm{H}_{3} \mathrm{CC}_{6} \mathrm{H}_{4}, \mathrm{H}_{7} \mathrm{C}_{3} \mathrm{OC}_{6} \mathrm{H}_{4}, 4-\mathrm{O}_{2} \mathrm{SC}_{6} \mathrm{H}_{4} ; \mathrm{X}=\mathrm{OCH}_{3}, \mathrm{NO}_{2}
$$

\section{Figure 31}

The reaction of 1-iodobutane or 2-bromo-1-(2,3-dihydro-1,4-benzodioxin-6-yl)-1-ethanone with triazoles 3 or 34 gave the corresponding alkyl sulfanyl derivatives 162 and 163 , respectively $^{37}$ (Figure 32 ).<smiles>[R1]n1c(SCCCCC)nnc1-c1cccc(OC)c1</smiles>

162

$$
\mathrm{R}^{1}=\mathrm{H}, \mathrm{Ph}
$$

Figure 32<smiles>[R]=C[R]c1ccc(OC)cc1</smiles> 
The reaction of chloroacetamide derivatives with 55 resulted in triazoles 164 and $\mathbf{1 6 5}^{\mathbf{1 7 4 , 1 7 5}}$ (Figure 33).<smiles>[R]C(Oc1ccccc1)c1nnc(SCC(=O)Nc2c(C)n(C)n(-c3ccccc3)c2=O)n1[R]</smiles>

164 $\mathrm{R}=\mathrm{CH}_{3} \mathrm{CH}_{2} ; \mathrm{C}_{6} \mathrm{H}_{5} ;$
$\mathrm{R}^{1}=\mathrm{H}, \mathrm{Cl} \mathrm{CH}_{3} ;$
$\mathrm{R}^{2}=\mathrm{Cl}, \mathrm{OCH}_{3}$<smiles>[R2]c1ccc2c(c1)C(NC(=O)CSc1nnc(C([R1])Oc3ccccc3)n1[R])CO2</smiles>

165

Figure 33

3-Benzylthio-4-alkyl/aryl-5-(4-aminophenyl)-4H-1,2,4-triazoles 166 were obtained by the reaction of 36 with benzyl chlorides in ethanolic sodium hydroxide ${ }^{176,177}$. Several 5-(1naphthylmethyl)- 4-aryl-s-triazol-3-thiols/ylthioglycolic acids $\mathbf{1 6 7}$ were synthesized as possible antiinflammatory agents ${ }^{178}$ (Figure 34 ).<smiles>[R]n1c(SCc2ccccc2)nnc1-c1ccc(N)cc1</smiles>

166, $\mathrm{R}=\mathrm{CH}_{3}, \mathrm{C}_{6} \mathrm{H}_{5}$<smiles>[R]n1c(SCC(=O)O)nnc1-c1cccc2ccccc12</smiles>

167, $\mathrm{R}=4-\mathrm{FC}_{6} \mathrm{H}_{4}, 2-\mathrm{H}_{3} \mathrm{C}-4-\mathrm{ClC}_{6} \mathrm{H}_{4}$ 2,4-, and 3,4-diClC ${ }_{6} \mathrm{H}_{3}$, 1-naphthyl, 2-naphthyl

Figure 34

A series of [(4,5-disubstituted-4H-1,2,4-triazol-3-yl)thio]alkanoic acids $\mathbf{1 6 8}$ were synthesized for their possible antiinflammatory activities ${ }^{54}$ (Figure 35).<smiles>[R]c1nnc(SC([R])OC)n1[R]</smiles>

168, $\mathrm{R}=\mathrm{CH}_{3} \mathrm{CH}_{2}, \mathrm{C}_{6} \mathrm{H}_{5}$;

$\mathrm{R}^{1}=4-\mathrm{ClC}_{6} \mathrm{H}_{4}, 4-$ pyridyl,

$3,4,5-$ tri- $\mathrm{CH}_{3} \mathrm{OC}_{6} \mathrm{H}_{2}$;

$\mathrm{R}^{2}=\mathrm{H}, \mathrm{CH}_{3}$

\section{Figure 35}


Glycosidation of 1,2,4-triazoline-5-thiones $\left(\mathrm{R}=\mathrm{H}, \mathrm{Me}, \mathrm{R}^{1}=\mathrm{H} ; \mathrm{R}=\mathrm{Ph}, 4\right.$-pyridyl, $\left.\mathrm{R}^{1}=\mathrm{Ph}\right)$ with $\mathrm{R}^{2} \mathrm{Br}\left(\mathrm{R}^{2}=\right.$ tetra- $O$-acetyl-D-glucopyranosyl,-galactopyranosyl,tri-o-acetyl-D-xylopyranosyl and L-arabinopyranosyl) in aq. acetone containing $\mathrm{KOH}$ gave 14-88\% yields of glycosides 169 which showed significant anti-inflammatory, analgesic, neurotropic, and antihypoxic activity. Deprotection of $169\left(\mathrm{R}=\mathrm{Me}, \mathrm{R}^{1}=\mathrm{H}, \mathrm{R}^{2}=\right.$ tri-o-acetyl- $\beta$-D-xylopyranosyl) with $\mathrm{NaOMe}$ in absolute methanol gave $68 \% 169$ (same $R, R^{1} ; R^{2}=-\beta$-D-xylopyranosyl) ${ }^{179}$. Also, 3,5disubstituted-1,2,4-triazole $170\left(\mathrm{R}=\mathrm{H}, \mathrm{Me}\right.$, or Et, $\left.\mathrm{R}^{1}=\mathrm{H}\right)$ derivatives and their $N$-glycosides $\left(\mathrm{R}^{1}\right.$ = tetraacetylglucos-amine or $\mathrm{N}$-acetylglucosamine) were prepared and tested for antiviral action against RNA-3 poliomyelitis in tissue culture ${ }^{180}$ (Figure 36).<smiles>[R]c1nnc([R7])n1[R1]</smiles>

169<smiles>[R]c1nc([As])n([R1])n1</smiles>

170

\section{Figure 36}

The bis[4-aryl-3-alkylthio-1,2,4-triazol-5-yl]alkanes 171 were prepared by the action of alkyl halides on bis[4-aryl-1,2,4-triazoline-5-thione-5-yl]alkanes $\mathbf{7 1}$ in aqueous sodium hydroxide $(5 \%)^{11,73,181}$ (Figure 37$)$.

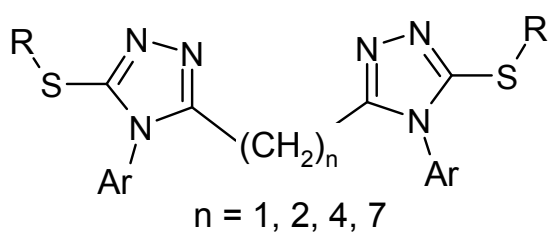

$$
\text { 171, } \begin{aligned}
\mathrm{R}= & \mathrm{CH}_{3}, \mathrm{CH}_{3} \mathrm{CH}_{2}, \mathrm{CH}_{3}\left(\mathrm{CH}_{2}\right)_{3}, \mathrm{CH}_{2}-\mathrm{CH}=\mathrm{CH}_{2}, \\
& \mathrm{CH}_{2} \mathrm{COOH}, \mathrm{C}_{6} \mathrm{H}_{5} \mathrm{CH}_{2}, 4-\mathrm{ClC}_{6} \mathrm{H}_{4}, \\
& 4-\mathrm{ClC}_{6} \mathrm{H}_{4} \mathrm{CH}_{2}, 4-\mathrm{BrC}_{6} \mathrm{H}_{4} \mathrm{CH}_{2}, 4-\mathrm{O}_{2} \mathrm{NC}_{6} \mathrm{H}_{4} \mathrm{CH}_{2}, \\
& 3,5-\mathrm{diO}_{2} \mathrm{NC}_{6} \mathrm{H}_{3}, 4-\mathrm{F}_{3} \mathrm{C}-2-\mathrm{O}_{2} \mathrm{NC}_{6} \mathrm{H}_{3}, \\
\mathrm{Ar}= & \mathrm{C}_{6} \mathrm{H}_{5}, 2-, \text { and } 3-\mathrm{H}_{3} \mathrm{CC}_{6} \mathrm{H}_{4}, 3-\mathrm{FC}_{6} \mathrm{H}_{4},
\end{aligned}
$$

Figure 37

The reaction of 73 with ethyl iodide in DMF at room temperature and in the presence of anhydrous potassium carbonate gave 5,5 -(1,4-phenylene)bis(3-ethylthio-4-phenyl-/benzyl-1,2,4triazole) $\mathbf{1 7 2}^{104}$ (Figure 38).<smiles>[Y10][Y17]=Cc1ccccc1</smiles>

\section{Figure 38}


5-Heteroarylthio-1,2,4-triazoles 173 were prepared by reaction of 1,2,4-triazole-5-thiones 3 or 26 with 2-bromopyridine or 2-ethoxy-6-nitro-9-chloroacridine. Compound $\mathbf{1 7 3}$ exhibited antiinflammatory, analgesic, neurotropic, and antihypoxic activity in rats and mice ${ }^{182,183}$ (figure 39 ).

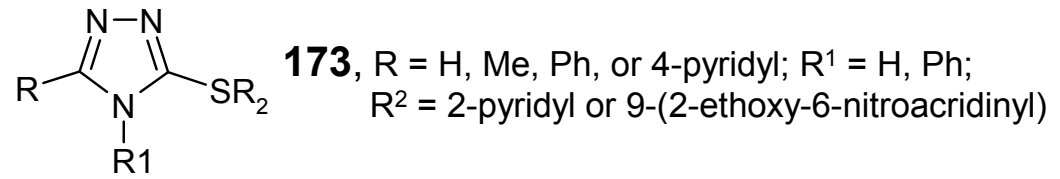

\section{Figure 39}

The alkylation of 75 with methyl iodide or 4-(chloroacetyl/ $\alpha$-chloropropionyl)-2,3dihydropyrazoles $\mathbf{1 7 5}$ in alkaline medium resulted in the production of the $S$-alkylated derivatives $174^{105}$ and $\mathbf{1 7 6}^{184}$ (Figure 40). Compound 176 were evaluated for in vitro antibacterial and antifungal activity ${ }^{184}$.<smiles>[R]c1ccc(-c2nnc(SC)n2C)o1</smiles>

$$
\begin{aligned}
\mathrm{R}= & \mathrm{CH}_{3}, \mathrm{C}_{4} \mathrm{H}_{9}, \mathrm{C}_{6} \mathrm{H}_{11}, \mathrm{C}_{6} \mathrm{H}_{5}, 4-\mathrm{H}_{3} \mathrm{CC}_{6} \mathrm{H}_{4}, 4-\mathrm{FC}_{6} \mathrm{H}_{4}, \\
& 4-\mathrm{ClC}_{6} \mathrm{H}_{4}, 4-\mathrm{BrC}_{6} \mathrm{H}_{4} ; \mathrm{R}^{1}=\mathrm{H}, \mathrm{CH}_{3}
\end{aligned}
$$

\section{Figure 40}

The alkylation of $\mathbf{1 2}$ or $\mathbf{7 6}$ with alkyl halide produced the $S$-allylated products $177^{44}$ and $\mathbf{1 7 8}^{54,106}$, respectively (Figure 41).<smiles>[R2]Sc1nnc(C2CCC(=O)N2[R1])n1[R]</smiles>

177, $\mathrm{R}=\mathrm{CH}_{3}, \mathrm{C}_{6} \mathrm{H}_{5} ; \mathrm{R}^{1}=\mathrm{H}, \mathrm{C}_{6} \mathrm{H}_{5} \mathrm{CH}_{2}$; $\mathrm{R}^{2}=\mathrm{CH}_{3}, \mathrm{CH}_{2} \mathrm{COOH}$,<smiles>[R1]Sc1nnc(-c2cc(C)on2)n1[R]</smiles>

178, $\mathrm{R}=\mathrm{CH}_{3}, \mathrm{CH}_{3} \mathrm{CH}_{2}, \mathrm{CH}_{3}-\left(\mathrm{CH}_{2}\right)_{3}-, \mathrm{C}_{6} \mathrm{H}_{11}, \mathrm{C}_{6} \mathrm{H}_{5}$ $\mathrm{R}^{1}=\mathrm{CH}_{3}, \mathrm{CH}_{2} \mathrm{CO}_{2} \mathrm{CH}_{3},\left(\mathrm{H}_{3} \mathrm{C}\right) \mathrm{CHCO}_{2} \mathrm{CH}_{3}$

\section{Figure 41}

Compounds $\mathbf{8 0}$ when treated with chloroacetic acid and benzyl chloride, yielded carboxymethylthio- 179 and benzylthio-5-(isomeric pyridyl)-1,2,4-triazoles $\mathbf{1 8 0}^{112,113,185}$ (Figure 42). 


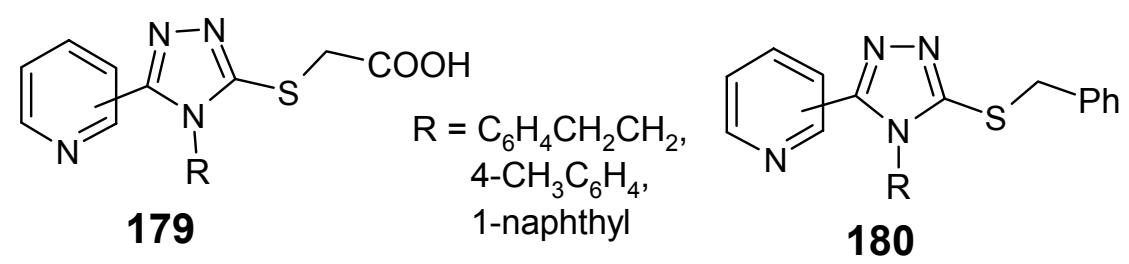

\section{Figure 42}

The methylation of compounds $\mathbf{8 2}$ or $\mathbf{8 4}$ with methyl iodide gave the corresponding $\mathbf{1 8 1} \mathbf{1}^{114}$ and $182^{116}$ (Figure 43).

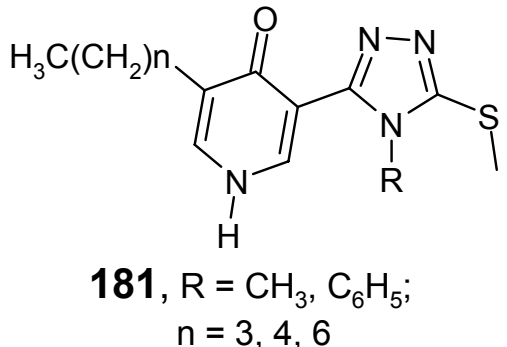<smiles>[R]n1nc(-c2nnc(SC)n2[R1])c(=O)cc1C</smiles>

\section{Figure 43}

Compound 86 was treated with diethylaminoethyl chloride hydrochloride and with 4-(2chloro-ethyl)morpholine hydrochloride in refluxing ethanol in the presence of anhydrous sodium acetate to give the corresponding $S$-alkylated products 183 and 184, respectively ${ }^{119}$ (Figure 44).<smiles>CCN(CC)CCSc1nnc(-c2cn(Cc3ccccc3)c3ccccc23)n1-c1ccccc1</smiles>

183<smiles>N#CCSc1nnc(-c2cn(Cc3ccccc3)c3ccccc23)n1-c1ccccc1</smiles>

184

\section{Figure 44}

The alkylation of 69 or 87 with ethyl iodide ${ }^{100}$ or methyl iodide ${ }^{53}$ or the hydrochlorides of $N, N$-disubstituted- $\beta$-chloroethylamines ${ }^{186}$ in an alkaline medium resulted in the production of 185 or 186 or 187 respectively (Figure 45 ). 


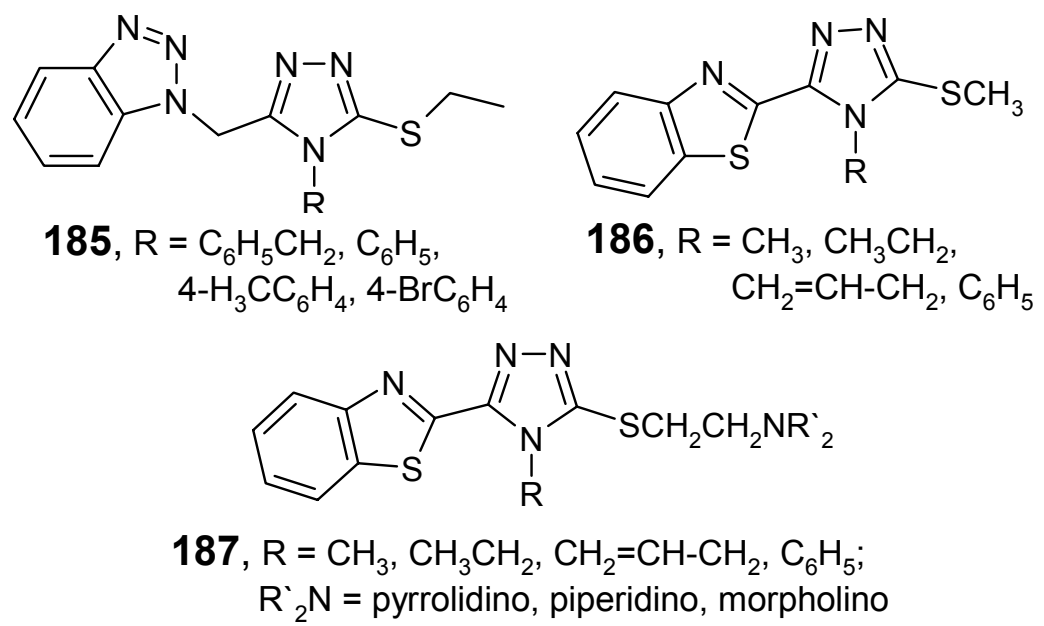

\section{Figure 45}

The $S$-alkylated derivatives $\mathbf{1 8 8}^{145}$ or $\mathbf{1 8 9}^{158}$ were synthesized from the reaction of the triazolothione 123 or 141 with $\alpha$-halo compounds, respectively (Figure 46).<smiles>[R]OC(=O)CSc1nnc2nc3sc(C([R])=O)c(N)c3c(-c3ccccc3)n12</smiles>

188, $R=O E t, M e ;$ $\mathrm{R}^{1}=\mathrm{OEt}, \mathrm{Ph}, \mathrm{NHPh}$<smiles>CSc1nnc2c(Cc3ccccc3)nn3c(=O)c4ccccc4nc3n12</smiles>

189

\section{Figure 46}

\subsection{Synthesis of monosulfides and disulfides}

When 3-methyl-1,2,4-triazole-5-thione (6), was allowed to react with diethyl azodicarboxylate, disulfide 190 was obtained ${ }^{187}$. Similarly, the disulfides 191 were prepared from $117^{140}$ (Figure 47).<smiles></smiles>

190<smiles>[R]n1cc(Cl)n2c(SSc3nnc4c(=O)n([R])cc(Cl)n34)nnc2c1=O</smiles>

191, $\mathrm{R}=\mathrm{CH}_{3}, \mathrm{C}_{6} \mathrm{H}_{5}$

\section{Figure 47}




\subsection{Synthesis of mannich base derivatives}

It has been found that the Mannich and double Mannich reaction starting from $s$-triazolo[3,4b]benzothiazol-3-thiol 128 to prepare some biologically active Mannich bases 192-194 ${ }^{149}$ (Figure 48).<smiles>Cc1ccc(NCn2nc3sc4ccccc4n3c2=S)cc1</smiles>

192<smiles></smiles>

193

Figure 48

\subsection{Synthesis of thiazolotriazoles}

The reaction of 1,2,4-1H-triazole 8 with $\alpha$-haloketones and with 1,2-dibromoethane leading to the formation of thiazolotriazole 195. ${ }^{188} 1,2,4$-Triazoline-3-thiones 104 upon treatment with sulfuric acid or bromine cyclize to derivatives of thiazolo[2,3-c][1,2,4]-triazole 196 and 197 respectively ${ }^{189}$ (Figure 49).<smiles>Clc1ccc(-c2ncc3n2CCS3)c(Cl)c1</smiles>

195

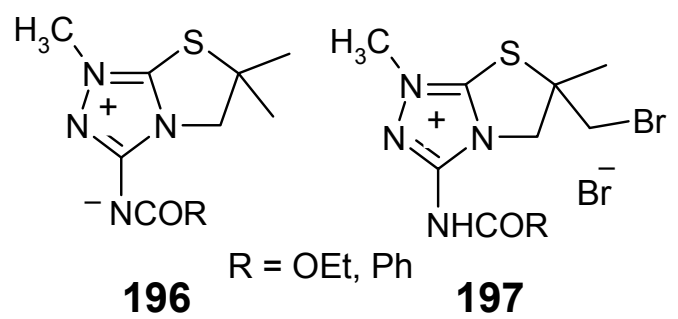

196

\section{Figure 49}

The facile and regioselective synthesis of 2-substituted-5-methylthiazolo[3,2-b]-1,2,4triazoles 199 proceeded via $\mathrm{H}_{2} \mathrm{SO}_{4}$ catalyzed cyclization of the corresponding (propynylthio)triazoles $\mathbf{1 9 8}^{190-194}$ (Figure 50). 


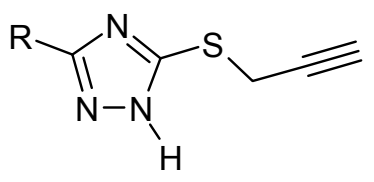

198<smiles>[R]c1nc2scc([N+](=O)[O-])n2n1</smiles>

199

$\mathrm{R}=\mathrm{H}, \mathrm{Me}, \mathrm{Ph}, \mathrm{MeC}_{6} \mathrm{H}_{4}, \mathrm{ClC}_{6} \mathrm{H}_{4}, \ldots$ etc.

\section{Figure 50}

The interaction of 4-allyl-1,2,4-triazole-3-thione $\mathbf{3 7}$ with iodine proceeds with the formation of a mixture of the iodo derivatives of thiazolotriazole and triazolothiazine which on elimination of $\mathrm{HI}$ gave the corresponding thiazolo[2,3-c]-1,2,4-triazole $\mathbf{2 0 0}$ and a mixture of 1,2,4-triazolo[3,4-b][1,3]thiazines 201 and 202 respectively ${ }^{195}$ (Figure 51).
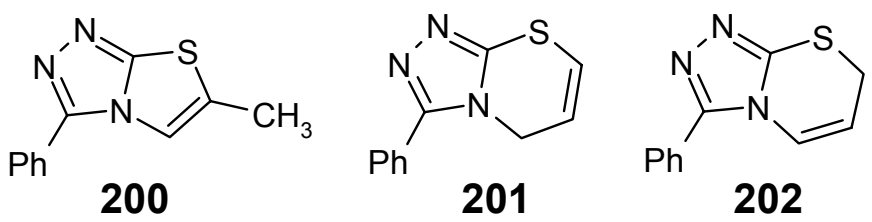

\section{Figure 51}

The triazoles 7 were condensed with either 3-(2-bromoacetyl)coumarins or with 3acetylcomarins using bromine in the presence of trichloro- $(N, N$-ethylene-bisaminobenzamide)lanthanum or samarium as a catalyst, followed by cyclization of the intermediate 3-alkyl-5-coumarinacyl-thio-s-triazoles 203 using PPA resulting in the formation of 3-alkyl-5-coumarinylthiazolo[3,2-b]triazoles $\mathbf{2 0 4}^{196}$ (Figure 52).<smiles>[R20]c1cc([R1])c2oc(=O)c(C(=O)CSc3nnc([R])[nH]3)cc2c1</smiles>

$$
\begin{aligned}
& \mathrm{R}=\mathrm{Me}, \mathrm{Et} ; \\
& \mathrm{R}^{1}=\mathrm{R}^{2}=\mathrm{H} \\
& \mathrm{R}^{1}=\mathrm{OMe}, \mathrm{R}^{2}=\mathrm{H} ; \\
& \mathrm{R}^{1}=\mathrm{H}, \mathrm{R}^{2}=\mathrm{Br} ; \\
& \mathrm{R}^{1}=\mathrm{R}^{2}=\mathrm{Br} ; \\
& \mathrm{R}^{1}=\mathrm{H}, \mathrm{R}^{2}=\mathrm{Cl} ; \\
& \mathrm{R}^{1}=\mathrm{H}, \mathrm{R}^{2}=\mathrm{Me}
\end{aligned}
$$<smiles>[R2]c1cc([R1])c2oc(=O)c(-c3csc4nc([R])nn34)cc2c1</smiles>

\section{Figure 52}

Cyclization of 1H-1,2,4-triazole-3-thiol $3(\mathrm{R}=\mathrm{H})$ with 3-chloro-2,4-pentadione in ethanol followed by bromination of the resulting 2-acetyl-3-methyl-1,2,4-triazolo[3,2-b]-thiazole with $\mathrm{Br}_{2}$ in $47 \%$ aq. $\mathrm{HBr}$, and refluxing 2-bromoacetyl-3-methyl-1,2,4-triazolo[3,2-b]thiazole with thiourea in ethanol afforded $\mathbf{2 0 5}^{197}$ (Figure 53). 
<smiles>Cc1c(-c2csc(N)n2)sc2ncnn12</smiles>

\section{Figure 53}

The 6-benzylidenethiazolo[3,2-b][1,2,4]triazole-5(6H)-ones 206 were synthesized by treatment of 9 with chloroacetic acid and substituted or non-substituted benzaldehydes in the presence of sodium acetate, acetic acid and acetic anhydride ${ }^{41,198,199}$ (Figure 54). Also, triazolothiazoles 208 was synthesized via cycloaddition of bis-hydrazonoyl chloride 207 with $\mathbf{3}^{200}$ (Figure 54).

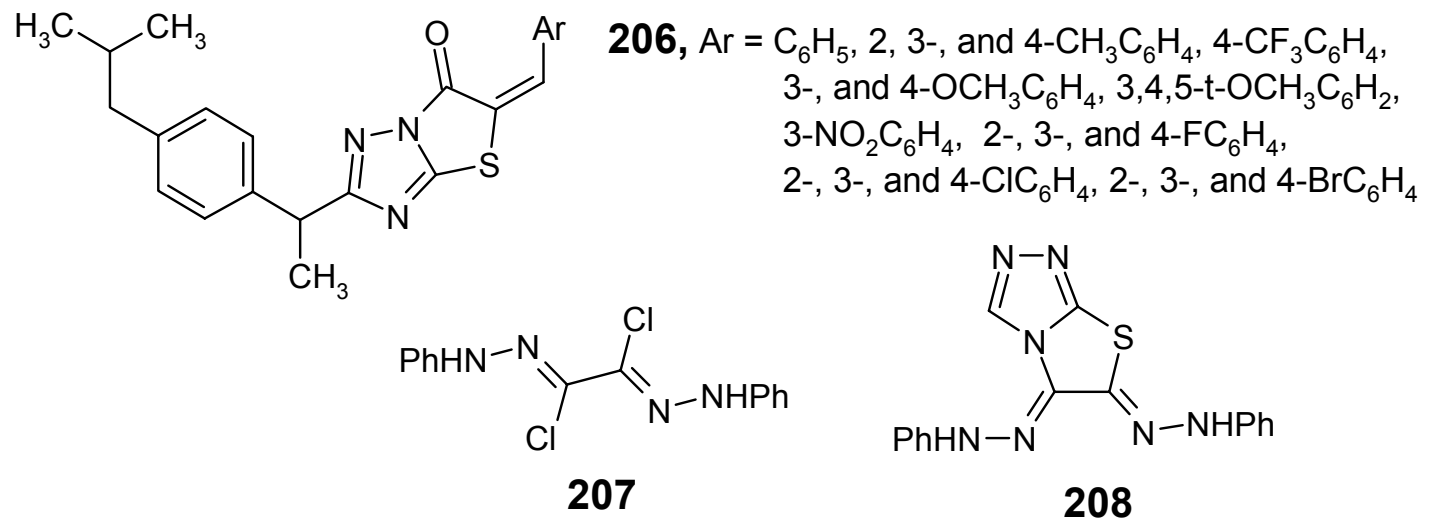

\section{Figure 54}

\subsection{Synthesis of triazolothiadiazoles}

The triazolothiadiazoles 209 have been synthesized by ring opening of various triazolo[3,4b] $[1,3,4]$ thiadiazoles $\mathbf{2 1 0}^{201}$ with $\mathbf{6}$ (Figure 55).<smiles>[R10]c1nnc(SCC(O)CN2C([R])Sc3nnc(C)n32)[nH]1</smiles>

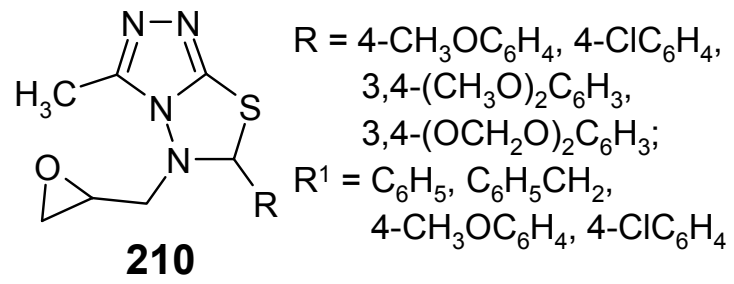

\section{Figure 55}




\subsection{Synthesis of triazolothiazines}

The condensation of triazolinethiones 3 or 7 with 3-aryl-2-propenoyl chlorides or 3-aryl-acryloyl chloride gave 5-aryl-5,6-dihydro-7H-[1,2,4]triazolo[5,1-b][1,3]thiazin-7-ones $\mathbf{2 1 1}^{202,203}$ (Figure 56).<smiles>[R]c1nc2n(n1)C(=O)CC([R])S2</smiles>

$$
\begin{aligned}
& \text { 211, R }= \mathrm{H}, \mathrm{C}_{6} \mathrm{H}_{5}, 4-\mathrm{H}_{3} \mathrm{COC}_{6} \mathrm{H}_{4}, 4-\mathrm{FC}_{6} \mathrm{H}_{4}, \mathrm{O}_{2} \mathrm{NC}_{6} \mathrm{H}_{4}, \\
& \text { 1-naphthyl, 1-adamantyl, }\left(\mathrm{C}_{6} \mathrm{H}_{5}\right)_{2} \mathrm{CH} \\
& \text { R1 = } \mathrm{C}_{6} \mathrm{H}_{5}, 4-\mathrm{H}_{3} \mathrm{COC}_{6} \mathrm{H}_{4}, 3,4\left(\mathrm{H}_{3} \mathrm{C}\right)_{2} \mathrm{C}_{6} \mathrm{H}_{3}, \\
& \text { 3-, and 4- } \mathrm{O}_{2} \mathrm{NC}_{6} \mathrm{H}_{4}, 4-\mathrm{ClC}_{6} \mathrm{H}_{4}, 4-\mathrm{FC}_{6} \mathrm{H}_{4}, \\
& \text { 1-naphthyl, 2-thienyl, 2-furyl }
\end{aligned}
$$

\section{Figure 56}

The condensation of $\mathbf{3}$ with 3-(4-[2-(chlorocarbonyl)ethenyl]phenyl)-acryloyl chloride in pyridine gave benzene-1,4-diaryl-bis-2-R-5-aryl-[1,2,4]triazolo[5,1-b]- [1,3]thiazin-7-ones $\mathbf{2 1 2}^{202}$ (Figure 57).<smiles>[R]c1nc2n(n1)C(=O)CC(c1ccc(C3CC(=O)n4nc([R])nc4S3)cc1)S2</smiles>

$$
\begin{aligned}
\text { 212, } \mathrm{R}= & \mathrm{H}, \mathrm{C}_{6} \mathrm{H}_{5}, 4-\mathrm{H}_{3} \mathrm{COC}_{6} \mathrm{H}_{4}, \\
& 4-\mathrm{FC}_{6} \mathrm{H}_{4}, \mathrm{O}_{2} \mathrm{NC}_{6} \mathrm{H}_{4}, \\
& \text { 1-naphthyl, 1-adamantyl, } \\
& \left(\mathrm{C}_{6} \mathrm{H}_{5}\right)_{2} \mathrm{CH}
\end{aligned}
$$

\section{Figure 57}

The 1,2,4-triazolo[5,1-b][1,3]thiazin-7-ones 213 have been first prepared by Peter et al. ${ }^{204}$ by cyclization of $\mathbf{3}$ with diethyl ethoxymethylenemalonate in fair to good yields. Also, Heindel et $\mathrm{al}^{205}$ have synthesized this heterocyclic system by condensation of $\mathbf{3}$ with methyl propionate, hydrolysis of the resulting $S$-acrylic esters to the corresponding $S$-acrylic acids 214, and subsequent cyclization to 213 or $\mathbf{2 1 5}$. The cyclization of 214 to 213 or 215 using thionyl chloride has also been reported as an independent synthesis (Figure 58).<smiles>[R]c1nc2sccc(=O)n2n1</smiles>

213, $\mathrm{R}=\mathrm{H}, \mathrm{CH}_{3}, \mathrm{C}_{6} \mathrm{H}_{5}$,<smiles>[R]c1nnc(SC=CC(=O)O)[nH]1</smiles>

214, $\mathrm{R}=\mathrm{H}, \mathrm{CH}_{3}, \mathrm{C}_{6} \mathrm{H}_{5}$,<smiles>[R]c1nnc2sccc(=O)n12</smiles>

215, $\mathrm{R}=\mathrm{H}, \mathrm{CH}_{3}, \mathrm{C}_{6} \mathrm{H}_{5}$,

Figure 58 
The 5-(perfluoroalkyl)triazole-3-thiols 3 reacts with methyl phenylpropynoate in boiling acetic acid or in boiling ethanol to form a mixture of the isomers 5H-1,2,4-triazolo[3,4b][1,3]thiazin-5-one 216 and 7H-1,2,4-triazolo[5,1-b][1,3]thiazin-7-one 217 in a total yield of $70-80 \%$ and a $10: 1$ ratio $^{206}$ (Figure 59).<smiles>[R]c1nnc2sc(-c3ccccc3)cc(=O)n12</smiles>

216<smiles>[R]c1nc2sc(-c3ccccc3)cc(=O)n2n1</smiles>

217

\section{Figure 59}

The addition of dimethylacetylene dicarboxylate or diethyl azodicarboxylate was obtained to 3 afforded in cycloadducts, which were identified as $\mathbf{2 1 8}^{187,207,208}$ or $\mathbf{2 1 9}^{187}$ (Figure 60). Compound 218 showed most remarkable anti-inflammatory activity in the carrageenan and serotonin induced diarrhea test ${ }^{208}$.

218, $\mathrm{R}=\mathrm{Me}, \mathrm{C}_{6} \mathrm{H}_{5}, 2-$, and $4-\mathrm{ClC}_{6} \mathrm{H}_{4}$,

2-, and $4-\mathrm{O}_{2} \mathrm{NC}_{6} \mathrm{H}_{4}$, $\left(\mathrm{H}_{3} \mathrm{C}\right)_{2} \mathrm{CHCH}_{2} \mathrm{C}_{6} \mathrm{H}_{3} \mathrm{CH}\left(\mathrm{CH}_{3}\right)-$<smiles>[R]c1nc2sc(C(=O)OC)cc(=O)n2n1</smiles><smiles>COC(=O)c1cc(=O)n2c(C)nnc2s1</smiles>

219

\section{Figure 60}

Reaction of triazolethione 3 with 1 mol PhC.tplbond.CCN or epibromohydrin gave $220^{163}$ or 3-hydroxy-1,2,4-triazolo[5,1-b]-1,3-thiazines $221^{171,209,210}$ (Figure 61).<smiles>[R]c1nc2sc(=N)cc(-c3ccccc3)n2n1</smiles>

220, $R=H, M e$<smiles>[R]c1nc2n(n1)CC(O)CS2</smiles>

221, $\mathrm{R}=\mathrm{Me}, \mathrm{Ph}$

\section{Figure 61}


The photo cyclization of substituted 1,2,4-triazole-3-thiones 222, under base-mediated conditions, afforded 1,2,4-triazolo[3,4-b]-1,3-(4H)-benzothiazines 223 with the desulfurization

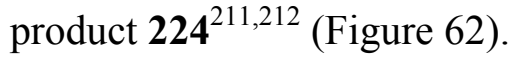<smiles>[R]c1nn([TlH])c(=S)n1C[Al]</smiles>

222<smiles>[R]c1nnc2n1Cc1ccccc1S2</smiles>

223<smiles>[R]c1nncn1C[Te]</smiles>

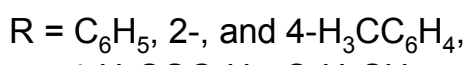
$4-\mathrm{H}_{3} \mathrm{COC}_{6} \mathrm{H}_{4}, \mathrm{C}_{6} \mathrm{H}_{4} \mathrm{CH}_{2}$; $\mathrm{Ar}=2-\mathrm{ClC}_{6} \mathrm{H}_{3}, 2-\mathrm{BrC}_{6} \mathrm{H}_{3}$

Figure 62

1,2,4-Triazolo[5 , 1 $\left.1^{\prime}: 2,3\right\}[1,3]$ thiazino[6,5-b]quinolines 225 have been synthesized by the reaction of 2-chloroquinoline-3-carboxaldehydes $\mathbf{2 2 6}$ with $\mathbf{3}$ and subsequent transformations of the hydroxyl group of $225\left(\mathrm{R}=\mathrm{OH}, \mathrm{R}^{1}=\mathrm{R}^{2}=\mathrm{H}\right)^{213}$ (Figure 63).<smiles>[R1]c1ccc2cc3c(nc2c1)Sc1nc([R])nn1C3[R]</smiles>

225<smiles>O=Cc1cc2ccccc2nc1Cl</smiles>

226

$\mathrm{R}=\mathrm{OH}, \mathrm{OEt}, \mathrm{OAc}, \mathrm{Cl}, \mathrm{OCH}_{2} \mathrm{CH}_{2} \mathrm{OH}, \mathrm{SCH}_{2} \mathrm{CH}_{2} \mathrm{OH}, \mathrm{NH}\left(\mathrm{CH}_{2}\right)_{3} \mathrm{Me}$;

$\mathrm{R}^{1}=\mathrm{H}, 6-$, 7-, 8-Me, 6-, 7-, 8-OMe, 7-, 8-Cl;

$\mathrm{R}^{2}=\mathrm{H}, \mathrm{CH}_{3}, \mathrm{CH}_{3} \mathrm{CH}_{2}$,

\section{Figure 63}

Similarly, synthesis of 1,2,4-triazolothiazinoquinoline 227-231 ${ }^{214-216}$ was described starting from 2-chloro-, or 4-chloro-3-(chloromethyl)quinoline and $\mathbf{3}$ (Figure 64). 
<smiles>[R]Oc1nc2n(n1)-c1nc3ccccc3cc1CS2</smiles><smiles>c1ccc2nc3c(cc2c1)Cn1cnnc1S3</smiles>

230<smiles>Cc1nc2ccccc2c2c1Cn1c(C)nnc1S2</smiles>

231

\section{Figure 64}

5H-1,2,4-Triazolo[5,1:2,3][1,3]thiazino[5,6-c]quinolin-5-ones $\mathbf{2 3 2}^{217}$ were prepared in 42$89 \%$ yield by the cyclocondensation of chloroquinoline carboxylates $\mathbf{2 3 3}$ with $\mathbf{3}$ in presence of $\mathrm{K}_{2} \mathrm{CO}_{3}$ in DMF (Figure 65).<smiles>[R]c1ccc2ncc3c(=O)n4nc([R])nc4sc3c2c1</smiles>

232
$\mathrm{R}=\mathrm{H}, \mathrm{OEt}, 8-\mathrm{Me}, 8-\mathrm{Et}$, 8-OMe, 8-Cl, 8- $\mathrm{F}, 8-\mathrm{NO}_{2}$, 9-Cl, 9-OMe, 10- $\mathrm{CHMe}_{2}$; $\mathrm{R}^{1}=\mathrm{H}, \mathrm{Me}$<smiles>[R]C=Cc1ccccc1Cl</smiles>

233

Figure 65

\subsection{Synthesis of triazolothiazepines}

Triazolobenzothiazepinones $\mathbf{2 3 4}{ }^{218}$ were synthesized in a regioselective manner via reaction of $\mathbf{3}$ with 2-chloromethyl-benzoyl chloride in good yields (Figure 66).<smiles>[R]C1=NN2C(=O)c3ccccc3CSC2N1</smiles>

234, $\mathrm{R}=\mathrm{H}, \mathrm{Me}, \mathrm{Ph}$

\section{Figure 66}




\subsection{Synthesis of triazolothiadiazines}

1,2,4-Triazolo[5,1-b][1,3,5]thiadiazin-7-ones $\mathbf{2 3 5}^{35}$ were obtained in good yield by the reactions of 3 with $N$-substituted- $N$-chloromethylcarbonyl chloride in the presence of potassium carbonate in DMF at room temperature (Figure 67).<smiles>[R]c1nc2n(n1)C(=O)N([R1])CS2</smiles>

$$
\begin{aligned}
\text { 235, } \mathrm{R} & =2-, \text { 3-, and 4- } \mathrm{FC}_{6} \mathrm{H}_{4}, 2,4-\mathrm{diCl}-5 \mathrm{FC}_{6} \mathrm{H}_{2} ; \\
\mathrm{R}^{1} & =\mathrm{C}_{2} \mathrm{H}_{5}, \mathrm{C}_{6} \mathrm{H}_{5}
\end{aligned}
$$

\section{Figure 67}

The di-Mannich reaction of 3 with aromatic amines and a formaldehyde solution in the presence of ethanol-HCl solution was used to produce the Mannich base namely 3,6disubstituted-1,2,4-triazolo[3,4-b][1,3,5]thiadiazines $236^{219}$ (Scheme 41). The resulting compounds 236 showed antibacterial activity against B. bob; S. aureus and E. coli at 800, 100 and 50 ppm concentrations ${ }^{219}$.<smiles>[R]c1n[nH]c(S)n1</smiles>

3 aq. $\mathrm{CH}_{2} \mathrm{O}(37 \%)$

$\mathrm{EtOH} / \mathrm{HCl}$

-<smiles>[R1]C=CCCC</smiles><smiles>[R]CCCSc1nnc([R])[nH]1</smiles$\mathrm{HCHO}$<smiles>[R]c1nnc2n1CN([R1])CS2</smiles><smiles>[R1]NCSc1nnc([R])n1CO</smiles>
or<smiles>[R]c1nnc(SCN([R])CO)[nH]1</smiles>

236, $\mathrm{R}=\mathrm{C}_{6} \mathrm{H}_{5}, 2-\mathrm{CH}_{3} \mathrm{OC}_{6} \mathrm{H}_{4}, 4-\mathrm{O}_{2} \mathrm{NC}_{6} \mathrm{H}_{4}$, $\mathrm{R}^{1}=\mathrm{C}_{6} \mathrm{H}_{5}, 4-\mathrm{CH}_{3} \mathrm{C}_{6} \mathrm{H}_{4}, 2-$, and 3- $\mathrm{NO}_{2} \mathrm{C}_{6} \mathrm{H}_{4}$

\section{Scheme 41}




\subsection{Miscellaneous reactions}

The 1,2,4-triazolo[3,4-c][1,2,4]triazole $237^{220}$ were prepared by reaction of 5-allylmercapto-3methyl-1,2,4-triazole with ethyl chloroglyoxalate p-tolylhydrazone was described (Figure 68).<smiles>CCC(=O)c1nn(-c2ccc(C)cc2)c2nnc(C)n12</smiles>

237

\section{Figure 68}

It has been found that two regioselective synthetic approaches for the $1 \mathrm{H}-[1,2,4]-$ triazolo[3,4-c][1,2,4]triazoles $\mathbf{2 4 3}$ via reaction of hydrazonoyl halide 238 with 3-methylthio-5phenyl-1,2,4-triazole (155) and base catalyzed cyclization of $N$-phenyl- $N$-(5-phenyl- $S$-triazol-3yl)thiohydrazides 239 were reported ${ }^{221}$. The structure of 243 was rationalized in terms of the initial formation of the amidrazones $\mathbf{2 4 0}$ as intermediates rather than $\mathbf{2 4 1}$ which cyclize in situ through elimination of methanethiol as soon as they are formed to give $\mathbf{2 4 3}$ as end products. Compound 243 exhibited antimicrobial activity against Escherichia coli, Bacillus subtilis and Pseudomonas aeruginosa (Figure 69). 


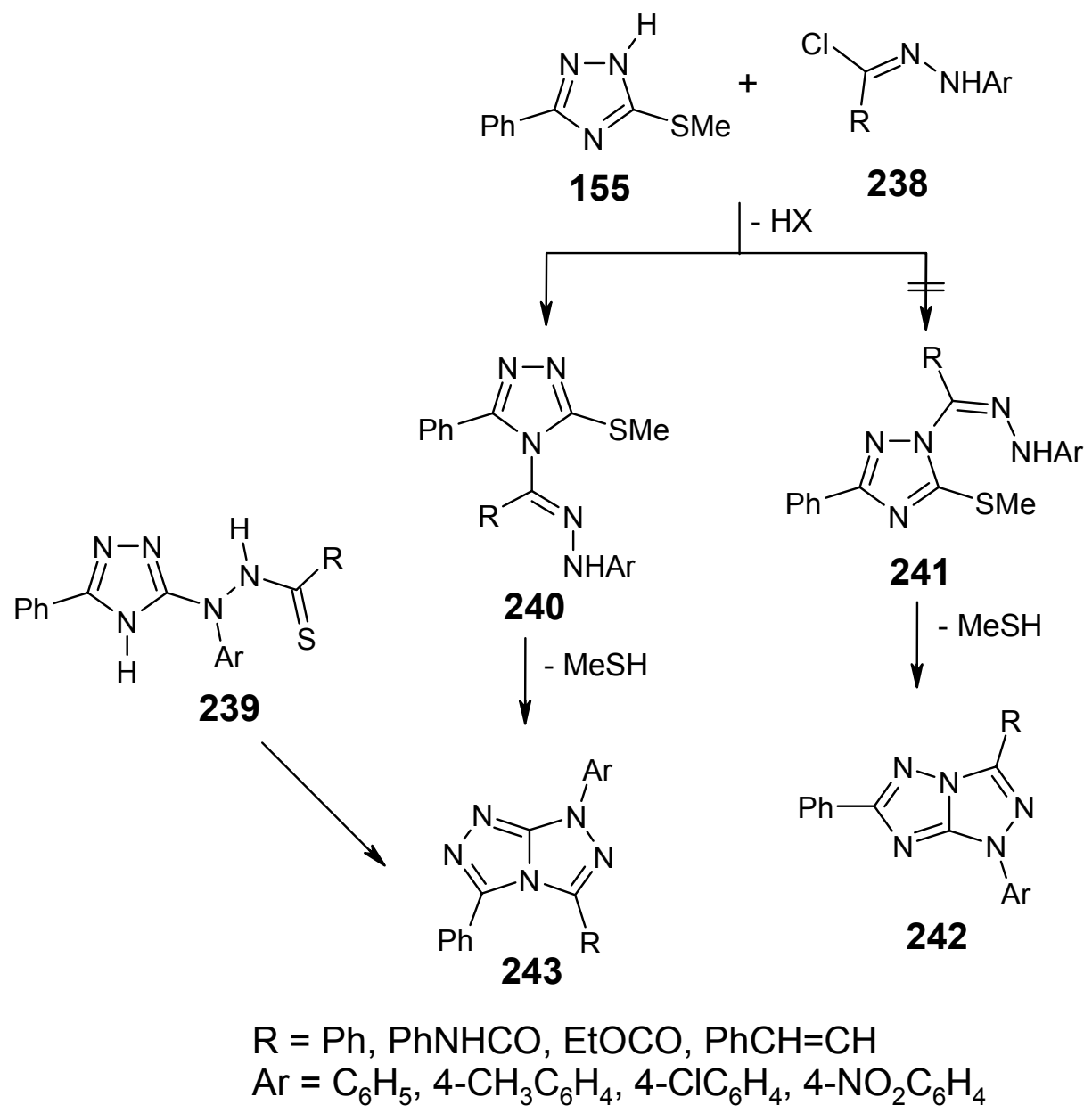

Figure 69

\section{Conclusions}

In this review the most important procedures used for the synthesis of mercapto-1,2,4-triazoles have been compiled and discussed. The mercapto/thioxo-1,2,4-triazole has proved to be a rich source of various heterocyclic compounds. Literature data published in the last 50 years have been included to help the reader to find information appropriate for the the chemistry of mercapto/thioxo-1,2,4-triazoles and their utility in heterocyclic synthesis.

\section{References}

1. Heindel, N. D.; Reid, J. R. J. Heterocycl. Chem. 1980, 17, 1087.

2. Holla, B. S.; Kalluraya, B.; Sridhar, K. R.; Drake, E.; Thomas, L. M.; Bhandary, K. K.; Levine, M.S. Eur. J. Med. Chem.1994, 29, 301. 
3. The Merck Index, 1996, $12^{\text {th }}$ Edn., Merck Co. Inc. Whitehouse Station.

4. Haber, J. Present status and perspectives on antimycoties with systematic effects, Cas. Lek. Cesk., 2001, 140, 596.

5. Brucato, A.; Coppola, A.; Gianguzza, S.; Provenzano, P. M. Bull. Soc. Ital. Biol. Sper. 1978, $54,1051$.

6. Coffen, D. L.; Fryer, R. I. US Pat., 1974, 3849 434. Chem. Abstr., 82, 730044v.

7. Shiroki, M.; Tahara, T.; Araki, K. Jap. Pat., 1975, 75100096; Chem. Abstr., 84, 59588k.

8. Povelitsa, F. D.; Gural, A. G. Antibiotiki Moscow, 1973, 18, 71. Chem. Abstr., 78, 93044.

9. Burch, H. A.; Smith, W. O. J. Med. Chem. 1966, 9, 405.

10. Foroumadi, A.; Mansouri, S.; Kiani, Z.; Rahmani, A. Eur. J. Med. Chem. 2003, 38, 851.

11. Ram, V. J.; Mishra, L.; Pandey, N. H.; Kushwaha, D. S.; Pieters, L. A. C.; Vlietinck, A. J. J. Heterocycl. Chem. 1990, 27, 351.

12. Ergenc, N.; Ilhan, E.; Ötük, G. Pharmazie 1992, 47, 59.

13. Kalyoncuoğlu, N.; Rollas, S.; Sür-Altiner, D.; Yegenoğlu, Y.; Anğ, Ö. Pharmazie 1992, 47, 796.

14. Rollas, S.; Kalyoncuoğlu, N.; Sür-Altiner, D.; Yegenoğlu, Y. Pharmazie 1993, 48, 308.

15. Mir, I.; Siddiqui, M. T.; Comrie, A. Tetrahedron 1970, 26, 5235.

16. Rudnicka, W.; Foks, H.; Janowiec, M.; Zwolska-Kwiek, Z. Acta Pol. Pharm. 1986, 43, 523; Chem. Abstr., 108, 131695 b.

17. Holla, B. S.; Veerendra, B.; Shivananda, M. K.; Poojary, B. Eur. J. Med. Chem. 2003, 38, 759.

18. Duran, A.; Dogan, H. N.; Rollas S., Farmaco 2002, 57, 559.

19. Yale, H. L.; Piala, J. J. J. Med. Chem. 1966, 9, 42.

20. Shah, M. H.; Mhasalkar, M. Y.; Palki, M. V.; Deliwala, C. V.; Sheth, U. K. J. Pharm. Sci. 1969, 58, 1398.

21. Mhasalkar, M. Y.; Shah, M. H.; Nikam, S. T.; Anantanarayanan, K. G.; Deliwala, C. V. J. Med. Chem. 1970, 13, 672.

22. Shaker, R. M.; Ali, A. A. Phosphorus, Sulfur Silicon Relat. Elem., (in press).

23. Hoggarth, E. J. Chem. Soc. 1949, 1163.

24. Jones, R. G.; Ainsworth, C. J. Am. Chem. Soc. 1955, 77, 1538.

25. Beyer, H.; Friedrich, C.; Busse, G. Liebigs Ann. Chem. 1960, 637, 135.

26. Potts, K. T.; Huseby, R. M. J. Org. Chem. 1966, 31, 3528.

27. Bourdais, J. Bull. Soc. Chim. France 1968, 3246.

28. Reid, J. R.; Heindel, N. D. J. Heterocycl. Chem. 1976, 13, 925.

29. Moskowitz, H.; Mignot, A.; Miocque, M. J. Heterocycl. Chem. 1980, 17, 1321.

30. Bermann, M. C.; Bonte, J. P.; Lesieur-Demarquilly, I.; Debaert, M.; Leieur, D.; Leinot, M.; Penoit, J.; Labrid, C. Eur, J. Med. Chem. 1982, 17, 85.

31. Eweiss, N. F.; A-Bahajaj, A.; Elsherbini, E. A. J. Heterocycl. Chem. 1986, 23, 1451.

32. Saramet, I.; Banciu, M. D.; Draghici, C. Rev. Roum. Chim. 1991, 36, 135.

33. Vershilov, S. V.; Popova, L. M.; Mungalov, V. E.; Ryabinin, N. A. Zh. Prikl. Khim. 1994, 
67, 1124. Chem. Abstr., 122, 265316.

34. Dieter, H. R.; Engel, J.; Klingler, K.; Kutscher, B.; Zelenyi, S. S.; Achterrath-Tuckermann, U.; Schmidt, J.; Metzenauer, P. Eur. Pat. Appl., 1994, EP584487 A2 19940302.

35. Liu, S.; Qian, X.; Song, G.; Chen, J.; Chen, W. J. Fluorine Chem. 2000, 105, 111.

36. Golovlyova, S. M.; Moskvichev, Yu. A.; Alov, E. M.; Kobylinsky, D. B.; Ermolaeva, V. V. Chem. Heterocycl. Compd. 2001, 37, 1102.

37. Labanauskas, L.; Udrenaite, E.; Gaidelis, P.; Brukstus, A. Farmaco 2004, 59, 255.

38. Siegfried, A.; Ernst, S. Ger. (East), 1985, pp 8. Chem. Abstr. 106, 5048.

39. Frederique, M.; Reve, M.; Geo, B. J. Heterocycl. Chem. 1984, 21, 1689.

40. Goswami, B. N.; Kataky, J. C. S.; Baruah, J. N. J. Heterocycl. Chem. 1984, 21, 1225.

41. Tozkoparan, B.; Gokhan, N.; Aktay, G.; Yesilada, E.; Ertan, M. Eur. J. Med. Chem. 2000, $35,743$.

42. Xu, P. F.; Sun, X. W.; Zhang, L. M.; Zhang, Z. Y. J. Chem. Res.(S) 1999, 170.

43. Mosher, W. A.; Toothill, R. B. J. Heterocycl. Chem. 1971, 8, 209.

44. Rigo, B.; Couturier, D. J. Heterocycl. Chem. 1989, 26, 1723.

45. Ozturk, S.; Akkurt, M.; Causiz, A.; Koparir, M.; Sekerci, M.; Heinemann, F. W.; Acta Cryst. E60, O425 (2004).

46. Koparir, Cetin, A.; Causiz, A.; Molecules 2005, 10, 475.

47. Ozturk, S.; Akkurt, M.; Causiz, A.; Koparir, M.; Sekerci, M.; Heinemann, F. W.; Acta Cryst, E60, O425 (2004).

48. Moustafa, O. S. Bull. Fac. Sci. Assiut Univ., 1995, 24(1)B, 289.

49. Moustafa, O. S. J. Chin. Chem. Soc. 2000, 47, 351.

50. Mekuskiene, G.; Gaidelis, P.; Vainilavicius, P. Pharmazie 1998, 53, 94.

51. Misra, U.; Hitkari, A.; Saxena, A. K.; Gurtu, S.; Shanker, K. Eur. J. Med. Chem.1996, 31, 629.

52. Ali, M. I.; Abdel-Fattah, A. M.; Hussain, S. M.; El-Reedy, A. M. J. Heterocycl. Chem. 1982, 19, 993.

53. Russo, F.; Santagati, M.; Pappalardo, G. Ann. Chim. 1972, 62, 351.

54. Sung, K.; Lee, A. R. J. Heterocycl. Chem. 1992, 29, 1101.

55. Parmar, S. S.; Gupta, A. K.; Singh, H. H.; Gupta, T. K. J. Med. Chem. 1972, 15, 999.

56. Cohen, V. I. J. Heterocycl. Chem. 1978, 15, 237.

57. Jaiswal, R. K.; Parmar, S. S.; Singh, S. P.; Barthwal, J. P. J. Heterocycl. Chem. 1979, 16, 561.

58. Bahel, S. C. J. Indian Chem. Soc. 1981, LVIII, 822.

59. Kane, J. M.; Dudley, M. W.; Sorensen, S. M.; Miller, F. P. J. Med. Chem. 1988, 31, 1235.

60. Kane, J. M.; Staeger, M. A.; Dalton, C. R.; Miller, F. P.; Dudley, M. W.; Ogden, A. M. L.; Kehne, J. H.; Ketteler, H. J.; McCloskey, T. C.; Senyah, Y.; Chmielewski, P. A.; Miller, J. A. J. Med. Chem. 1994, 37, 125.

61. Kane, J. M.; Dalton, C. R.; Staeger, M. A.; Huber, E. W. J. Heterocycl. Chem. 1995, 32, 183. 
62. Dobosz, M.; Pitucha, M.; Wujec, M. Acta Polon. Pharm. 1996, 53, 31.

63. Milcent, R.; Nguyen, T. H. J. Heterocycl. Chem. 1986, 23, 881.

64. Lin, Y. I.; Petty, S. R.; Lovell, F. M.; Perkinson, N. A.; Lang, Jr., S. A. J. Heterocycl. Chem. 1980, 17, 1077.

65. Katica, C. R.; Vesna, D.; Vlado, K.; Dora, G. M. J. Heterocycl. Chem. 2003, 40, 905.

66. Fulop, F.; Semega, E.; Dombi, G.; Bernath, G. J. Heterocycl. Chem. 1990, 27, 951.

67. Nath, T. G.; Husain, S.; Srivasan, V. P. Indian J. Chem. 1977, 15B, 341.

68. Wang, Z. Y.; You, T. P.; Shi, H. J.; Shi, H. X. Goading Xuaxiao Huaxue Xuebao 1997, 18(4), 550; Chem. Abstr., 127, 95265.

69. Sarvá, M. C.; Romeo, G.; Guerrera, F.; Siracusa, M.; Salerno, L.; Russo, F.; Cagnotto, A.; Goegan, M.; Mennini, T. Biorg. Med. Chem. 2002, 10, 313.

70. Strzemecka, L. Pol. J. Chem. 1983, 57, 567.

71. C. R. Katica, D. Vesna, K. Vlado, G. M. Dora, Molecules 6, 815 (2001).

72. Kömürcü, S. G.; Rollas, S.; Ulgen, M.; Gorrod, J. W.; Cevikbas, A. Boll. Chim. Fr. 1995, 134, 375.

73. Ram, V. J.; Dube, V.; Pieters, L. A. C.; Vlietinck, A. J. J. Heterocycl. Chem. 1989, 26, 625.

74. Dogan, H. N.; Rollas, S.; Erdeniz, H. Farmaco 1989, 53, 462.

75. Schenone, S.; Bruno, O.; Ranise, A.; Bondavalli, F.; Filippelli, W.; Rossi, F.; Falcone, G. Farmaco 1998, 53, 590.

76. Dobosz, M.; Pachuta-Stec, A. Acta Polon. Pharm. 1995, 52, 103.

77. Cansiz, A.; Koparir, M.; Demirdag, A. Molecules 2004, 9, 204.

78. Iqbal, R.; Rama, N. H.; Ahmed, N.; Zamani, K.; Ebrahim, S.; Iqbal, N. Indian J. Chem. 1998, 37B, 506.

79. Kothari, P. J.; Kishore, V.; Stenberg, V. I.; Parmar, S. S. J. Heterocycl. Chem. 1978, 15, 1101.

80. Omar, A. M. M. E.; AboulWafa, O. M. J. Heterocycl. Chem. 1984, 21, 1419.

81. Gupta, A. K. S.; Bajaj, O. P.; Chandra, U. J. Indian Chem. Soc. 1978, 55, 962.

82. Suman, S. P.; Bahel, S. C. J. Indian Chem. Soc. 1980, 57, 420.

83. Pathak, R. B.; Bahel, S.C. J. Indian Chem. Soc. 1981, 58, 420.

84. Triredi, S.; Kubarate, H.; Parekh, H. Indian J. Chem. 1994, 33, 295.

85. Vashi, S.; Mehta, S.; Shah, V. H. Indian J. Chem. 1996, 35, 11.

86. Palaska, E.; Sahin, G.; Kelicen, P.; Durlu, N. T.; Altinok, G. Farmaco 2002, 57, 101.

87. Ahluwalia, V. K.; Mann, R. R.; Bala, S. Indian J. Chem. 1989, $28 B, 247$.

88. Gupta, A. K. S.; Hajela, K.; Bhattacharya, T.; Ahmad, S.; Shanker, K. J. Heterocycl. Chem. 1983, 20, 507.

89. Wujec, M.; Pitucha, M.; Dobosz, M.; Kosikowska, U.; Malm, A. Acta Pharm. 2004, 54, 251.

90. Ahmad, R.; Zia-Ul-Haq, M.; Jabeen, R.; Duddeck, H. Turk. J. Chem. 1996, 186.

91. Demirbas, N.; Karaoglu, S. A.; Demirbas, A.; Sancak, K. Eur. J. Med. Chem. 2004, 39, 793.

92. Kothari, P. J.; Stenberg, V. I.; Singh, S. P.; Parmar, S. S.; Zoellner, R. W. J. Heterocycl. 
Chem. 1980, 17, 637.

93. Kothari, P. J.; Singh, S. P.; Parmar, S. S.; Stenberg, V. I. J. Heterocycl. Chem. 1980, 17, 1393.

94. Smicius, R.; Jakubkiene, V.; Burbuliene, M. M.; Mikalainyte, A.; Vainilavicius, P. J. Chem. Res. (S) 2002, 170.

95. Varvaresou, A.; Papastaikoudi, T. S.; Tsotinis, A.; Kakoulidou, A. T.; Vamvakides, A. Farmaco 1998, 53, 320.

96. Sawney, S. N.; Singh, J.; Bansal, O. P. Indian J. Chem. 1975, 13, 804.

97. Murthy, G. R.; Reddy, V. M. Sulfur Lett. 1988, 7, 171.

98. Youssef, K. M.; El-Meligil, S. Egypt. J. Pharm. Sci. 1989, 30, 455.

99. Rani, B. R.; Bhalerao, U. T.; Rahman, M. F. Indian J. Chem. 1990, 29B, 995.

100.El-Khawass, S. M.; Habib, N. S. J. Heterocycl. Chem. 1989, 26, 177.

101.Young, R. W.; Wood, K. H. J. Am. Chem. Soc. 1955, 77, 400.

102.Ram, V. I.; Mishra, L.; Pandey, H. N.; Mishra, S. Ind. J. Chem. 1979, 18B, 203.

103.Ram, V. I. Arch. Pharm. (Weinheim) 1979, 312, 147.

104. Shaker, R. M.; Mahmoud, A. F.; Abdel-Latif, F. F. Phosphorus, Sulfur Silicon Relat. Elem. 2005, 180, 397.

105.Benjamin, L. E.; Snyder, Jr., H. R. J. Heterocycl. Chem. 1976, 13, 1115.

106. Shafiee, A.; Naimi, E.; Mansobi, P.; Foroumadi, A.; Shekari, M. J. Heterocycl. Chem. 1995, $32,1235$.

107.Rostom, S. A. F.; Shalaby, M. A.; El-Demellawy, M. A. Eur. J. Med. Chem. 2003, 38, 959.

108. Mahajan, R. N.; Havaldar, F. H.; Fernandes, P. S. J. Indian Chem. Soc. 1991, 68, 245.

109.Chu, C. H.; Hui, X. P.; Zhang, Y.; Zhang, Z. Y.; Li, Z. C.; Liao, R. A. J. Chin. Chem. Soc. 2001, 48, 121.

110.Lagorce, J. F.; Comby, F.; Buxeraud, J.; Raby. C. Eur. J. Med. Chem. 1992, 27, 359.

111.Iqbal, R.; Zamani, K.; Rama, N. H.; Turk. J. Chem. 1996, $20,295$.

112.Zamani, K.; Faghihi, K.; Sangi, M. R.; Zolgharnein, J. Turk. J. Chem. 2003, 27, 119.

113.Zamani, K.; Faghihi, K.; Tofighi, T.; Shariatzadeh, M. R. Turk. J. Chem. 2004, $28,95$.

114.Balogh, M.; Hermecz, I.; Meszaros, Z.; Pusztay, L. J. Heterocycl. Chem. 1980, 17, 175.

115.Mekuskiene, G.; Tumkevicius, S.; Vainilavicius, P. J. Chem. Res. (S) 2002, 213.

116.Zou, X.; Jin, G. J. Heterocycl. Chem. 2001, 38, 993.

117. Shutske, G. M. J. Heterocycl. Chem. 1981, 18, 1017.

118.Kus, C.; Ayhan-Kilcigil, G.; Can, B. E.; Iscan, M. Arch. Pharmacal. Res. 2004, 27, 156.

119. Farghaly, A. A. H. J. Chin. Chem. Soc. 2004, 51, 147.

120.Shiba, S. A.; El-Kamary, A. A.; Shaban, M. E.; Atia, K. S. Indian J. Chem. 1997, $36 b, 36$.

121.Kurasawa, Y.; Muramatsu, M.; Yamazaki, K.; Tajima, S.; Okamoto, Y.; Takada, A. J. Heterocycl. Chem. 1986, 23, 633.

122.Kurasawa, Y.; Muramatsu, M.; Yamazaki, K.; Tajima, S.; Okamoto, Y.; Takada, A. J. Heterocycl. Chem. 1986, 23, 959.

123.Kurasawa, Y.; Muramatsu, M.; Yamazaki, K.; Tajima, S.; Okamoto, Y.; Takada, A. J. 
Heterocycl. Chem. 1986, 23, 1379.

124.Kurasawa, Y.; Muramatsu, M.; Yamazaki, K.; Tajima, S.; Okamoto, Y.; Takada, A. J. Heterocycl. Chem. 1986, 23, 1391.

125.Noto, R.; Buccheri, F.; Cusmano, G.; Gruttadauria, M.; Werber, G. J. Heterocycl. Chem. 1991, 28, 1421.

126.Noto, R.; Meo, P. L.; Gruttadauria, M.; Werber, G. J. Heterocycl. Chem. 1999, 36, 667.

127.Noto, R.; Meo, P. L.; Gruttadauria, M.; Werber, G. J. Heterocycl. Chem. 1996, 33, 863.

128. Michelangelo, G.; Francesco, B.; Silvestre, B.; Giuseppe, C.; Renato, N.; Giuseppe, W. J. Heterocycl. Chem. 1992, 29, 233.

129. Silvestre, B.; Michelangelo, G. Tetrahedron 2000, 56, 999.

130.Buccheri, F.; Cusmano, G.; Gruttadauria, M.; Noto, R.; Werber, G. J. Heterocycl. Chem. 1997, 34, 1447.

131. Vicentini, C. B.; Manfrini, M.; Veronese, A. C.; Guarneri, M. J. Heterocycl. Chem. 1998, $35,29$.

132.Kane, J. M. Synthesis 1987, 912.

133.Elmoghayar, M. R. H.; Elnagdi, M. H.; Ibrahim, M. K. A.; Sallam, M. M. M. Helv. Chim. Acta 1977, 60, 2171.

134.Ersan, S.; Richter, C.; Hobert, A.; Mariam, G. G.; Schulzey, K. J. Heterocycl. Chem. 1995, 32, 275.

135.Ershov, A. Yu. Khim. Geterotsikl. Soedin. 2002, 828.

136.Ershov, A. Yu.; Koshmina, N. V.; Mokeev, M. V.; Gribanov, A. V. Chem. Heterocycl. Compd. 2003, 39, 1257.

137.Raphael, E.; Joshua, C. P.; Koshy, L. Indian J. Chem. 1989, 28B, 635.

138. Shaker, R. M. Phosphorus, Sulfur Silicon Relat. Elem. 2003, 178, 1175.

139.El-Reedy, A. M.; Ayyad, A. O.; Ali, A. S. J. Heterocycl. Chem. 1989, 26, 313.

140.Bhattacharya, B. K. J. Heterocycl. Chem. 1986, 23, 113.

141.Lovelette, C. A. J. Heterocycl. Chem. 1979, 16, 555.

142. Styskala, J.; Slouka, J.; Wiedermannova, I.; Bednar, P. J. Heterocycl. Chem. 2003, 40, 805.

143.Baraldi, P. G.; El-Kashef, H.; Farghaly, A.; Vanelle, P.; Fruttarolo, F. Tetrahedron 2004, 60, 5093.

144.Badr, M. Z. A.; El-Sherief, H. A.; El-Naggar, G. M.; Mahgoub, S. A. J. Heterocycl. Chem. 1984, $21,471$.

145. Geies, A. A. J. Chem. Res. (S) 1998, 290.

146. Abdel Moneam, M. I. Monatsh. Chem. 2004, 135, 45.

147.Simó, M.; Csámpai, A.; Harmat, V.; Barabás, O.; Magyarfalvy, G. Tetrahedron 2001, 57, 7191.

148.Bower, J. D.; Doyle, F. P. J. Chem. Soc. 1957, 1-3, 727 ().

149.Zhang, Y.; Hui, X. P.; Dai, C. F.; Zhang, Z. Y.; Xu, P. F. Chin. J. Chem. 2002, 20, 381.

150.Hassan, N. A. Molecules 2000, 5, 826.

151.Li, Z.; Meng, Q. Q.; Wang, Q. R.; Tao, F. G. Chin. J. Chem. 2004, 22, 290. 
152.Effi, Y.; Lancelot, J. C.; Rault, S.; Robba, M. J. Heterocycl. Chem. 1983, 20, 913.

153. Nagai, S. I.; Takemoto, S.; Ueda, T.; Mizutani, K.; Uozumi, Y.; Tokuda, H. J. Heterocycl. Chem. 2001, 38, 1097.

154.Oganisyan, A. Sh.; Noravyan, A. S.; Grigoryan, M. Zh. Chem. Heterocycl. Compd. 2004, $40,75$.

155.Abdel-Latif, F. F.; Shaker, R. M.; Mahgoub, S. A.; Badr, M. Z. A. J. Heterocycl. Chem. 1989, 26, 769.

156.Eid, M. M.; El-Zanaty, A.; Lbrahim, Y. A. J. Heterocycl. Chem. 1990, 27, 591.

157.Basyouni, W. M.; Hosni, H. M.; El-Bayouki, K. A. M. J. Chem. Res.(S), 1997, 452; (M), 2771.

158.El-Agrody, A. M.; Abd El-Latif, M. S.; El-Hady, N. A.; Fakery, A. H.; Bedair, A. H. Molecules 2001, 6, 519.

159.Fahmy, S. M.; Kandeel, E. M.; Elsayed, E-F. R.; Elnagdi, M. H. J. Heterocycl. Chem. 1978, 15, 1291.

160. Rahmiye, E.; Gulgun, A. FABAD Farmasotik Bilimler Dergisi (1976-2000) 1990, 15(1), 1; Chem. Abstr., 114, 101840.

161.Trzhitsinskaya, B. V.; Rudakova, E. V.; Afonin, A. V.; Aksenov, V. P. Izv. Akad. Nauk SSSR Ser. Khim., 1986, 7, 1674; Chem. Abstr. 106, 196334.

162. Vereshchagin, L. I.; Bol'Shedvorskaya, R. L.; Pavlova, G. A.; Alekseeva, N. V. Khim. Geterotsikl. Soedin. 1979, 11, 1552; Chem. Abstr., 92, 215349.

163.Abramova, N. D.; Trzhtsinskaya, B. V.; Skvortsov, Y. A.; Mal'Kina, A. G.; Albanov, A. I.; Skvortsova, G. G. Khim. Geterotsikl. Soedin. 1982, 8, 1051; Chem. Abstr. 97, 216085.

164.Cherkovskaya, L. G.; Knysh, E. G.; Rogul'chenko, G. K.; Drogovoz, S. M.; Sal'nikova, S. I.; Steblyuk, I. N. Farmatsevtichnii Zhurnal (Kiev) 1989; 5, 67; Chem. Abstr. 112, 198233.

165.Yang, G. F.; Lu, R. J.; Fei, X. N. Chin. J. Chem. 2000, 18, 435.

166.Radics, U.; Liebscher, J. J. Heterocycl. Chem. 1993, 30, 1061.

167.Trzhtsinskaya, B. V.; Skvortsova, G. G.; Mansurov, Y. A.; Buchin, P. I.; Viderker, M. G. Khim. -Farm. Zh. 1982, 16(12), 1466; Chem. Abstr. 98, 143339.

168.Ersan, S.; Nacak, S.; Berkem, R.; Özden, T. Arzneim. -Forsch. Drug Res. 1997, 47, 963.

169.Heravi, M. M.; Tajbakhsh, M. J. Chem. Res. 1998, 488.

170.Upadhyaya, P.; Surendra Nath, T. G.; Srinvasan, V. R. Synthesis 1978, 288.

171.Trzhtsinskaya, B. V.; Kositsyna, E. I.; Pertsikov, B. Z.; Rudakova, E. V.; Voronov, V. K.;

Shvortsova, G. G. Khim. Geterotsikl. Soedin. 1987, 2, 271; Chem. Abstr. 107, 198211.

172.Korotkikh, N. I. Z. Org. Khim. 1989, 25(7), 1533; Chem. Abstr. 112, 118729.

173.Salerno, L.; Siracusa, M.; Guerrera, F.; Romeo, G.; Pittalá, V.; Modica, M.; Mennini, T.; Russo, F. ARKIVOC 2004, 312.

174.Gülhan, T. Z.; Meltem, S.; Fatma, S. K.; Kevser, E. Eur, J. Med. Chem. 2001, 36, 685.

175.Turan-Zitouni, G.; Kaplancikli, Z. A.; Ozdemir, A. Farmaco 2002, 57, 573.

176.Ram, V. G.; Vidyottama, D.; Pieters, L. A.; Vlietinck, A. J. J. Heterocycl. Chem. 1989, 26, 625. 
177.Kücükgüzel, I.; Kömürcü, S. G.; Rollas, S. Symposium Abstracts Book, $4^{\text {th }}$ International Symposium on Pharmaceutical Sciences 1995, June 27-30, Ankara, Turkey; 0-13.

178.Kothari, P. J.; Mehlhoff, M. A.; Singh, S. P.; Parmar, S. S.; Stenberg, V. I. J. Heterocycl. Chem. 1980, 17, 1369.

179.Knysh, E. G.; Mazur, I. A.; Zimenkovskii, B. S.; Stets, V. R.; Komissarento, N. F. Farmatsevtichnii Zhurnal (Kiev) 1987, 5, 35; Chem. Abstr. 109, 129494.

180.Mevlut, E.; Seyhan, E. Ankara Univ. Eczacilik Fak. Dergisi 1984, 14(1), 81; Chem. Abstr. 104, 61528.

181.Ram, V. J.; Vlietinck, A. J. J. Heterocycl. Chem. 1988, 25, 253.

182.Knish, E. G.; Gurko, I. V.; Stets, V. R.; Mazur, I. A. Farmatsevtichnii Zhurnal (Kiev) 1983, 2, 64; Chem. Abstr. 99, 38421.

183.Knysh, E. G.; Mazur, I. A.; Stets, V. R.; Savenkova, N. N. Farmatsevtichnii Zhurnal (Kiev) 1986, 2, 49; Chem. Abstr. 105, 72087.

184.Ulusoy, N.; Kücükbasmaci, Ö.; Kiraz, M.; Yeğenoğlu, Y. Monatsh. Chem. 2003, 134, 465.

185.Iqbal, B.; Zamani, K.; Rama, N. H. Turk. J. Chem. 1996, 20, 295.

186.Russo, F.; Santagati, M. Bull. Chim. Farm. 1982, 121, 159.

187.Gaetano, G.; Maria, N.; Bianca, C.; Also, P.; Carmelo, L. R. J. Heterocycl. Chem. 1991, 28, 325.

188. Goswami, B. N.; Kataky, J. C. S.; Baruah, J. N. J. Heterocycl. Chem. 1986, 23, 1439.

189.Ersan, S.; Jelonek, S.; Sieler, J.; Schulze, K. Tetrahedron 1996, 52, 791.

190.Potts, K. T.; Hussain, S. J. Org. Chem. 1971, 36, 10.

191.Heravi, M. M.; Tajbakhsh, M.; Ramezanian, N. Indian J. Heterocycl. Chem. 1998, 7, 309.

192.Heravi, M. M.; Tajbakhsh, M.; Mohajerani, B.; Riahifar, N. Indian J. Heterocycl. Chem. 1999, 8, 247.

193.Heravi, M. M.; Tajbakhsh, M.; Rahimizadeh, M.; Davoodnia, A. Indian J. Heterocycl. Chem. 1999, 8, 341.

194.Heravi, M. M.; Tajbakhsh, M.; Rahimizadeh, M.; Davoodnia, A.; Aghapoor, K. Synth. Commun. 1999, 29, 4417.

195.Shmygarev, V. I.; Kim, D. G. Chem. Heterocycl. Compd. 2004, 40, 1077.

196. Vardhan, A. V.; Kumar, R. V.; Rao, R. R. Indian J. Chem. 1999, 38B, 18.

197.Yoo, H. Y.; Chung, K. J.; Chai, J. P.; Chang, M. S.; Kim, S. G.; Choi, W. S.; Kang, D. P.; Kim, Y. H.; Kim, Y. H.; Woo, T. W.; Paek, J. H.; Seo, K. H. PCT Int. Appl. 1997, 26 pp; Chem. Abstr. 126, 186096.

198. Tozkoparan, B.; Akgün, H.; Ertan, M.; Rübseman, K. Arch. Pharm. 1995, 328, 169.

199. Tozkoparan, B.; Ayhan, G. K.; Ertan, R.; Ertan, M.; Kelicen, P.; Demirdamar, R. Arzneim. Forsch. Drug Res. 1999, 49, 1006.

200.Dawood, K. M.; Elwan, N. M. J. Chem. Res. 2004, 264.

201. Gupta, R.; Sudan, S.; Meng, V.; Kachroo, P. L. Indian J. Chem. 1996, 35B, 621.

202. Kudryavtsev, A. A.; Lozinskii, M. O. Russ. J. Org. Chem. 2004, 40, 232.

203. Britsun, V. N.; Lozinskii, M. O. Chem. Heterocycl. Compd. 2004, 40, 1092. 
204.Peter, C. J.; Peter, O. H.; Trevor, K. J. J. Chem. Soc.,Perkin Trans I 1980, 7, 1352.

205.Heindel, N. D.; Reid, J. R. J. Org. Chem. 1980, 45, 2479.

206.Danilkina, N. A.; Vershilov, S. V.; Ganina, M. B.; Mikhailov, L. E.; Ivin, B. A. Russ. J. Gen. Chem. 2004, 74, 472.

207.Heravi, M. M.; Beheshtiha, Y. S.; Oskooie, H. A.; Nami, N. Indian J. Heterocycl. Chem. 1999, 8, 245.

208.Birsen, T.; Goknur, A.; Erdern, Y. Farmaco 2002, 57, 145.

209.Heravi, M. M.; Rabiee, M.; Bolurtchian, M. Indian J. Heterocycl. Chem. 1998, 8, 165.

210.Heravi, M. M.; Rabiee, M.; Bolurtchian, M. Indian J. Heterocycl. Chem. 2000, 10, 153.

211. Senthilvelan, A.; Ramakrishnan, V. T. Tetrahedron Lett. 2002, 43, 5119.

212. Senthilvelan, A.; Thirumalai, D.; Ramakrishnan, V. T. Tetrahedron 2004, 60, 851.

213.Ferenc, K.; Zoltan, C.; Zoltan, S. Heterocycles 1992, 34, 1711.

214.Ferenc, K.; Zoltan, S. Heterocycl. Commun. 1995, 1, 297.

215.Ferenc, K.; Jozef, J.; Zoltan, S. J. Heterocycl. Chem. 1997, 34, 1275.

216.Korodi, F.; Jeko, J.; Szabo, Z. J. Heterocycl. Chem. 1997, 34, 1275.

217.Zoltan, S.; Ferenc, K. Synth. Commun. 1990, 20, 2473.

218. Bakavolia, M.; Davoodnia, A.; Rahimizadeh, M.; Heravib, M. M. J. Chem. Res (S) 2002, 4, 178.

219. Wang, Z.; You, T.; Shi, H.; Shi, H. Molecules 1996, 1, 89.

220.Lazrak, F.; Ahabchane, N. H.; Keita, A.; Essassi, E. M.; Pierrot, M. Indian J. Chem. 2002, $41 B, 821$.

221. Shawali, A. S.; Abdallah, M. A.; Abbas, I. M.; Eid, G. M. J. Chin. Chem. Soc. 2004, 51, 351.

\section{Biographical Sketch}

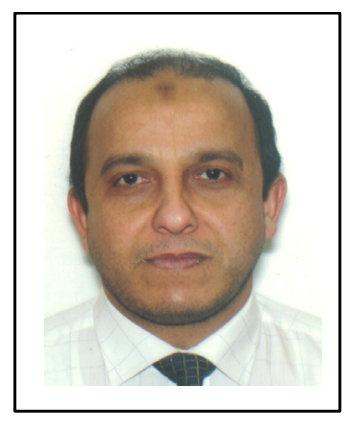

Raafat M. Shaker was born in 1963 in El-Minya City, Egypt. He obtained a B. Sc. in 1985, a M. Sc. in 1989 and Ph.D. (Organic Synthesis) in 1994 from Chemistry Department, Faculty of Science, El-Minya University, Egypt. During his Ph. D., he worked, for two years, under supervision of Prof. Dr. H. H. Otto, Albert-Ludwigs-Freiburg University, Germany. He was promoted to the rank of associated professor in 2000. His research is focusing on synthetic 
organic chemistry. His general plan is to investigate new methods for the synthesis of the versatile and hitherto unreported heterocyclic compounds of expected biological activity. He got a research fellowship from Deutsche Forschungs-gemeinschaft (DFG) with Prof. Hartmut Laatsch, Department of Organic Chemistry, University of Goettingen, Germany. He also obtained a grant by the Royal Society of Chemistry with Dr. Grahame Mackenzie, Department of Chemistry, University of Hull, England. 